\title{
Orbifold Singularities,
}

\section{Lie Algebras of the Third Kind (LATKes), and Pure Yang-Mills with Matter}

\author{
Tamar Friedmann ${ }^{1,2}$ \\ Massachusetts Institute of Technology \\ Cambridge, MA 02139, USA
}

\begin{abstract}
We discover the unique, simple Lie Algebra of the Third Kind, or LATKe, that stems from codimension 6 orbifold singularities and gives rise to a new kind of YangMills theory which simultaneously is pure and contains matter. The root space of the LATKe is 1-dimensional and its Dynkin diagram consists of one point. The uniqueness of the LATKe is a vacuum selection mechanism.
\end{abstract}

\footnotetext{
${ }^{1}$ E-mail: tamarf@mit.edu

${ }^{2}$ Current address: tamarf@pas.rochester.edu
} 


\title{
The World in a Point?
}

Blow-up of $\mathbf{C}^{3} / \mathbf{Z}_{3} \mid$ Dynkin diagram of the LATKe

\author{
Pure Yang-Mills with matter
}




\section{Introduction}

The interpretation of codimension 4 orbifold singularities as ADE gauge theories, which arose within string theory in the mid 90's [1,2], has been extended to the case of M-theory compactifications, where codimension 4 orbifold singularities in $G_{2}$ spaces were also interpreted as ADE gauge theories [3, 4, 5]. Further orbifolding the already-singular $G_{2}$ spaces led us to the first manifestation via M-theory of Georgi-Glashow grand unification: from an $A_{4}$ singularity of the $G_{2}$ space, an $S U(5)$ gauge theory broken by Wilson lines precisely to the gauge group of the standard model $S U(3) \times S U(2) \times U(1)$ arose naturally, with no extraneous gauge fields $[6,7,8]$. A precise relation between the energy scale of grand unification $\left(M_{G U T}\right)$ and certain volumes inside the $G_{2}$ space was also obtained $[8,9]$.

In the process of constructing the $G_{2}$ spaces, orbifold singularities of codimension 6 arose as well [7]. However, there was no analog of the interpretation of a codimension 4 singularity as an ADE gauge theory for the case of codimension 6. We set out to find such an analog.

To our delight and surprise, we discover far more than we expected, both mathematically speaking and physically speaking.

On the math side, we introduce a new set of relations, which we call the Commutator-Intersection Relations, that illuminate the connection between codimension 4 singularities and Lie algebras. These relations pave the way to construct Lie Algebras of the Third Kind, or LATKes, a kind of algebras that arise from codimension 6 orbifold singularities. We also learn and prove the existence and uniqueness of a simple LATKe.

On the physics side, we discover a new kind of Yang-Mills theory, called "LATKe Yang-Mills," which arises from the LATKe. Unlike any known YangMills theory, the LATKe Yang-Mills theory in its purest form automatically contains matter. We also propose that the uniqueness of the simple LATKe is a vacuum selection mechanism. The selected vacuum theory is an $S U(2) \times S U(2)$ 
gauge theory with matter in the $(2,2)$ representation, and the corresponding singularity is $\mathbf{C}^{3} / \mathbf{Z}_{3}$. The algebra $\mathfrak{s u}(2) \times \mathfrak{s u}(2)$ is protected by the LATKe from being broken. The selected singularity $\mathbf{C}^{3} / \mathbf{Z}_{3}$ is one of those which arose in the $G_{2}$ spaces of [7], and which at the time we put on hold in anticipation of the outcome of this investigation.

\section{The Codimension 4 Case}

In this section, we review the correspondence between codimension 4 orbifold singularities and ADE Lie algebras, introduce the Commutator-Intersection Relations, and review the relation between physical interactions on the one hand and commutators and intersections on the other hand.

\subsection{Du Val-Artin correspondence}

The interpretation of codimension 4 orbifold singularities as ADE gauge theories is mathematically rooted in the work of Du Val and of Artin [10, 11, 12], who pointed out a correspondence between certain singularities and their blowups on the one hand and certain Lie algebras on the other hand.

Before we state the correspondence, we provide below the necessary ingredients.

The singularities in question are those that appear at the origin of $\mathbf{C}^{2}$ under the orbifold action of finite discrete subgroups of $S U(2)$. These subgroups, denoted $\Gamma$, had been classified as early as 1884 by F. Klein [13]. They consist of the cyclic groups $\mathbf{Z}_{n}$, also denoted $A_{n-1}$; the binary dihedral groups $\mathbf{D}_{n}$; and three "exceptional" groups: the binary tetrahedral T, binary octahedral $\mathbf{O}$, and binary icosahedral $\mathbf{I}$, also denoted $E_{6}, E_{7}$, and $E_{8}$, respectively. Such a classification is known as an ADE classification.

Each of these subgroups of $S U(2)$ has a natural action on $\mathbf{C}^{2}$. For example, 
$\mathbf{Z}_{n}$ is generated by the $S U(2)$ matrix

$$
\left(\begin{array}{cc}
e^{2 \pi i / n} & 0 \\
0 & e^{-2 \pi i / n}
\end{array}\right)
$$

and acts on $(x, y) \in \mathbf{C}^{2}$ via the two-dimensional representation

$$
(x, y) \longmapsto\left(e^{2 \pi i / n} x, e^{-2 \pi i / n} y\right) .
$$

The singularity at the origin of $\mathbf{C}^{2}$ is analyzed by blowing up: the singular space is replaced by a smooth manifold that looks just like $\mathbf{C}^{2} / \Gamma$ everywhere except at the origin, and the origin itself is replaced by a smooth space of real dimension 2. This 2-dimensional space, known as the exceptional divisor, turns out to be a union of intersecting $\mathbf{P}^{1}$ s, or 2 -spheres $\mathbf{S}^{2}$.

In the ambient four-dimensional space, the intersection of any two $\mathbf{P}^{1}$ s is zero dimensional, i.e. it is a set of points. Counting those points gives an intersection number. The set of all intersection numbers forms the intersection matrix of the exceptional divisor, which we denote $\left\{I_{i j}\right\}$. The indices $i j$ run from 1 to $b_{2}$, where $b_{2}$ is the number of independent 2-cycles in the exceptional divisor.

As it happens, the intersection matrix of the exceptional divisor of $\mathbf{C}^{2} / \Gamma$ is equal to the negative of the Cartan matrix of the A, D, or E Lie algebra corresponding to $\Gamma$ :

$$
C_{i j}=-I_{i j} .
$$

In addition, the exceptional divisor itself becomes the Dynkin diagram of the corresponding Lie algebra when we replace each component $\mathbf{P}^{1}$ of the exceptional divisor by a node and connect a pair of nodes by an edge when their corresponding $\mathbf{P}^{1}$ 's intersect; the components of the exceptional divisor therefore correspond to the simple positive roots of the Lie algebra. For example, when $\Gamma=\mathbf{Z}_{3}$, the exceptional divisor is two intersecting $\mathbf{P}^{1}$ 's and the Dynkin diagram consists of two connected nodes:

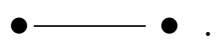


Now we are ready to state the Du Val-Artin correspondence:

$$
\begin{aligned}
\left\{\begin{array}{c}
\text { Exceptional divisor } \\
\text { of blow-up of } \mathbf{C}^{2} / \Gamma
\end{array}\right\} & =\left\{\begin{array}{l}
\text { Dynkin diagram of } \\
\text { ADE Lie algebra }
\end{array}\right\} \\
\left\{\begin{array}{c}
\text { Intersection matrix of } \\
\text { exceptional divisor } \\
I_{i j}
\end{array}\right\} & =\left\{\begin{array}{c}
\text { Negative Cartan matrix } \\
\text { of ADE Lie algebra } \\
-C_{i j}
\end{array}\right\}
\end{aligned}
$$

\subsection{Commutator-Intersection Relations}

Here we rephrase the Du Val-Artin correspondence as a set of relations between commutators of the Lie algebra and intersection numbers of the exceptional divisor, as follows.

A complex simple Lie algebra is generated by $k$ triples $\left\{X_{i}, Y_{i}, H_{i}\right\}_{i=1}^{k}$ with their commutators determined by the following relations:

$$
\begin{aligned}
& {\left[H_{i}, H_{j}\right]=0 ;} \\
& {\left[X_{i}, Y_{j}\right]=\delta_{i j} H_{j} ;} \\
& {\left[H_{i}, X_{j}\right]=C_{i j} X_{j} ;} \\
& {\left[H_{i}, Y_{j}\right]=-C_{i j} Y_{j} ;} \\
& \operatorname{ad}\left(X_{i}\right)^{1-C_{i j}}\left(X_{j}\right)=0 ; \\
& \operatorname{ad}\left(Y_{i}\right)^{1-C_{i j}}\left(Y_{j}\right)=0 .
\end{aligned}
$$

Here, the $H_{i}$ form the Cartan subalgebra, the $X_{i}$ are simple positive roots, the $Y_{i}$ are simple negative roots, $k$ is the rank of the Lie algebra, $C_{i j}$ is the Cartan matrix, and $\operatorname{ad}\left(X_{i}\right)(A)=\left[X_{i}, A\right]$. These equations are the familiar Chevalley-Serre relations.

Now recall from equations (2.3) and (2.6) that $C_{i j}=-I_{i j}$. If we replace 
$C_{i j}$ in equations (2.7) by $-I_{i j}$, we get a new set of relations:

$$
\begin{aligned}
& {\left[H_{i}, H_{j}\right]=0 ;} \\
& {\left[X_{i}, Y_{j}\right]=\delta_{i j} H_{j} ;} \\
& {\left[H_{i}, X_{j}\right]=-I_{i j} X_{j} ;} \\
& {\left[H_{i}, Y_{j}\right]=I_{i j} Y_{j} ;} \\
& \operatorname{ad}\left(X_{i}\right)^{1+I_{i j}}\left(X_{j}\right)=0 ; \\
& \operatorname{ad}\left(Y_{i}\right)^{1+I_{i j}}\left(Y_{j}\right)=0 .
\end{aligned}
$$

These relations demonstrate that the intersection numbers of the exceptional divisor completely determine the commutators of the corresponding Lie algebra.

This role of the intersection numbers in the structure of the Lie algebra will be central for us in what follows, and we will refer to the relations (2.8) as the Commutator-Intersection Relations, or the CI Relations.

\subsection{Interactions, commutators, and intersections}

Physically speaking, there are relations between interactions and commutators, and interactions and intersections.

Here, we explain roughly how ADE gauge fields arise from the codimension 4 singularities $[14,15,16]$. First, Kaluza Klein reduction of 3-form C-fields on 2 -cycles of the exceptional divisor provides the gauge fields corresponding to the Cartan subalgebra. Second, D2-branes wrapped on 2-cycles provide the "charged" gauge fields, forming the rest of the Lie algebra.

For example, in the $\mathbf{C}^{2} / \mathbf{Z}_{2}$ case, where the exceptional divisor is a single $\mathbf{P}^{1}$, three fields arise: a $3-$ form field reduced on the $\mathbf{P}^{1}$, which gives rise to the Cartan element denoted $A_{\mu}$; and D2-branes wrapped on the $\mathbf{P}^{1}$, which give rise to two oppositely charged particle states denoted $W^{+}$or $W^{-}$depending on orientation. 
The interaction among these fields can be pictorialized in the following way:
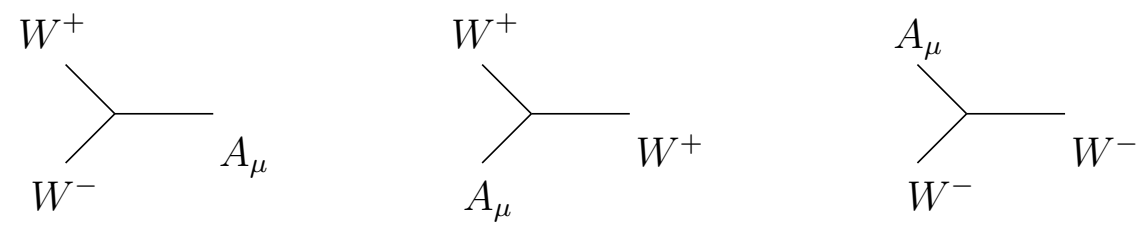

The three interactions depicted above, $W^{+} W^{-} \rightarrow A_{\mu}, W^{+} A_{\mu} \rightarrow W^{+}$, and $A_{\mu} W^{-} \rightarrow W^{-}$, all arise from the same interaction vertex and are related to each other by CPT symmetry.

These interactions can then be manifested as Lie algebra commutators:

$$
\left[W^{+}, W^{-}\right]=A_{\mu}, \quad\left[W^{+}, A_{\mu}\right]=W^{+}, \quad\left[A_{\mu}, W^{-}\right]=W^{-} .
$$

These commutators form precisely the $\mathfrak{s u}(2)$ Lie algebra.

In addition to the manifestation of interactions as commutators, when the singularities are such that the exceptional divisor contains more than a single cycle, the intersections between the cycles can be interpreted as interactions between fields wrapped or reduced on those cycles [14].

\section{Lie Algebras of the Third Kind (LATKes)}

In this section we define the algebraic objects that are related to codimension 6 orbifold singularities in a way analogous to the relation between Lie algebras and codimension 4 singularities.

Recall from Section 2.2 that in the correspondence between codimension 4 singularities and Lie algebras, the intersection numbers of pairs of cycles in the exceptional divisor determine the Lie algebra commutators via the CI Relations (equations 2.8). In particular, the intersection numbers enter the following commutators:

$$
\begin{aligned}
& {\left[H_{i}, X_{j}\right]=-I_{i j} X_{j}} \\
& {\left[H_{i}, Y_{j}\right]=I_{i j} Y_{j} .}
\end{aligned}
$$


Also recall the physics interpretation of commutators and intersections as interactions between two fields (Section 2.3).

Now, for codimension $2 n$ singularities for any $n$, the components of the exceptional divisor are $(2 n-2)$-cycles, and the intersection of a pair of those has dimension

$$
\operatorname{dim}\left(C_{1} \cap C_{2}\right)=\operatorname{dim} C_{1}+\operatorname{dim} C_{2}-2 n=2 n-4
$$

Therefore, for codimension 6 orbifolds, the components of the exceptional divisor are 4-cycles, and the intersection of any pair $C_{1}, C_{2}$ of 4-cycles does not yield a number but a two-dimensional space:

$$
\operatorname{dim}\left(C_{1} \cap C_{2}\right)=4+4-6=2 .
$$

However, the intersection of three 4-cycles in a six dimensional ambient space is zero-dimensional, yielding intersection numbers with three indices: $I_{i j k}$. On the physics side, these triple intersections should lead to interactions among three fields.

Bringing together all the above leads us to a generalization of the CI Relations to the codimension 6 case. Equations (3.1) become commutators of three objects:

$$
\begin{aligned}
& {\left[A_{i}, B_{j}, X_{k}\right]=-I_{i j k} X_{k}} \\
& {\left[A_{i}, B_{j}, Y_{k}\right]=I_{i j k} Y_{k} .}
\end{aligned}
$$

At this stage, we do not yet know what the $A_{i}, B_{j}, X_{k}$, or $Y_{k}$ are. However, equation (3.4) provides the fundamental ingredient in the algebraic objects we have been searching for: a commutator with three entries. It is now clear that the sought-after algebraic objects are the following natural generalizations of Lie algebras:

Definition 3.1 A Lie algebra of the third kind (a "LATKe") $\mathfrak{L}$ is a vector space equipped with a commutator of the third kind, which is a trilinear anti-symmetric map

$$
[\cdot, \cdot, \cdot]: \Lambda^{3} \mathfrak{L} \rightarrow \mathfrak{L}
$$


that satisfies the Jacobi identity of the third kind:

$\left[X, Y,\left[Z_{1}, Z_{2}, Z_{3}\right]\right]=\left[\left[X, Y, Z_{1}\right], Z_{2}, Z_{3}\right]+\left[Z_{1},\left[X, Y, Z_{2}\right], Z_{3}\right]+\left[Z_{1}, Z_{2},\left[X, Y, Z_{3}\right]\right]$

for $X, Y, Z_{i} \in \mathfrak{L}$.

We will refer to the commutator of the third kind as a "LATKe commutator", and to the Jacobi identity of the third kind as the "LATKe Jacobi identity." The LATKe Jacobi identity is simply a generalization of the standard Jacobi identity, which says that the adjoint action is a derivation.

For codimension $2 n$ singularities, it is $n$-fold intersections of $(2 n-2)-$ cycles that give intersection numbers. So the CI Relations for that case have commutators of $n$ objects, leading to the following generalization of a LATKe:

Definition 3.2 A Lie algebra $\mathfrak{L}$ of the n-th kind (a "LAnKe") is a vector space equipped with a commutator of the $n$-th kind, which is an n-linear, totally antisymmetric map

$$
[\cdot, \cdot, \quad, \cdot]: \wedge^{n} \mathfrak{L} \rightarrow \mathfrak{L}
$$

that satisfies the Jacobi identity of the $n$-th kind:

$$
\left[X_{1}, \ldots X_{n-1},\left[Z_{1}, \ldots Z_{n}\right]\right]=\sum_{i=1}^{n}\left[Z_{1}, \ldots,\left[X_{1}, \ldots X_{n-1}, Z_{i}\right], \ldots Z_{n}\right]
$$

for $X_{i}, Z_{j} \in \mathfrak{L}$.

Before we go any further, we should satisfy ourselves that LATKes actually exist. Therefore, we now construct an example.

The algebra $\mathfrak{L}_{3}$ Recall that in the codimension 4 case, each component of the exceptional divisor corresponds to a node in the Dynkin diagram of the corresponding Lie algebra. Therefore, each component corresponds to a simple root of the Lie algebra. Furthermore, the simplest singularity is $\mathbf{C}^{2} / \mathbf{Z}_{2}$, whose blow-up is a single $\mathbf{P}^{1}$, and the simplest (non-abelian) Lie algebra is the corresponding $\mathfrak{s u}(2)$. 
It is reasonable to presume that similarly, the simplest example of a LATKe would correspond to the codimension 6 orbifold singularity with the simplest exceptional divisor. Consider the singularity $\mathbf{C}^{3} / \mathbf{Z}_{3}$, where the action of a generator $\epsilon$ of $\mathbf{Z}_{3}$ is given by

$$
\epsilon:(x, y, z) \longmapsto(\epsilon x, \epsilon y, \epsilon z) \text {, where } \epsilon^{3}=1,(x, y, z) \in \mathbf{C}^{3}
$$

The blow-up of this singularity is a single $\mathbf{P}^{2}$ (see Appendix A). The cycle $\mathbf{P}^{2}$ corresponds to a node in the yet-to-be-defined Dynkin diagram of $\mathfrak{L}_{3}$. That node, in turn, should correspond to a yet-to-be-defined "root" of $\mathfrak{L}_{3}$.

We must now define the notion of a root for LATKes. For standard Lie algebras, a root $\alpha$ is in the dual space of the Cartan subalgebra $\mathfrak{h}$ :

$$
\alpha: \mathfrak{h} \longrightarrow \mathbf{C}
$$

So, to define a root for $\mathfrak{L}$, we must first define a Cartan subalgebra for $\mathfrak{L}$.

In the standard Lie algebra case, one can think of the Cartan subalgebra as a set of operators acting on the Lie algebra $\mathfrak{g}$ : for a root vector $X_{\alpha} \in \mathfrak{g}$ and for $H \in \mathfrak{h}$, we have

$$
H \quad: \quad X_{\alpha} \longmapsto\left[H, X_{\alpha}\right]=\alpha(H) X_{\alpha} .
$$

For a LATKe $\mathfrak{L}$, there is no natural action of a subalgebra. However, if $\mathfrak{h}_{\mathfrak{L}} \subset \mathfrak{L}$ is a subalgebra of $\mathfrak{L}$ (a subspace closed under the commutator), then $\Lambda^{2} \mathfrak{h}_{\mathfrak{L}}$ does act on $\mathfrak{L}$ naturally: if $H_{1}, H_{2} \in \mathfrak{h}_{\mathfrak{L}}$ and $X \in \mathfrak{L}$, then $H_{1} \wedge H_{2} \in \Lambda^{2} \mathfrak{h}_{\mathfrak{L}}$ acts on $\mathfrak{L}$ via:

$$
H_{1} \wedge H_{2} \quad: \quad X \longmapsto\left[H_{1}, H_{2}, X\right]
$$

We may now define $\mathfrak{h}_{\mathfrak{L}} \subset \mathfrak{L}$ the same way it is defined for Lie algebras:

Definition 3.3 A Cartan subalgebra $\mathfrak{h}_{\mathfrak{L}}$ of $\mathfrak{L}$ is a maximal commuting subalgebra of $\mathfrak{L}$ such that $\Lambda^{2} \mathfrak{h}_{\mathfrak{L}}$ acts diagonally on $\mathfrak{L}$.

Finally, following the standard Lie algebra case, we have 
Definition 3.4 Let $\mathfrak{L}$ be a LATKe and let $\mathfrak{h}_{\mathfrak{L}}$ be a Cartan subalgebra of $\mathfrak{L}$. A root $\alpha$ of $\mathfrak{L}$ is a map in the dual space of $\Lambda^{2} \mathfrak{h} \mathfrak{L}$ :

$$
\alpha: \Lambda^{2} \mathfrak{h}_{\mathfrak{L}} \longrightarrow \mathbf{C}
$$

Now we may also define a generalization for Dynkin diagrams:

Definition 3.5 A Dynkin diagram of $\mathfrak{L}$ is a graph with a vertex for each simple positive root of $\mathfrak{L}$.

By "positive," we imply that we have chosen a direction in $\left(\Lambda^{2} \mathfrak{h}_{\mathfrak{L}}\right)^{*}$ and ordered the roots, as is done for conventional Lie algebras. Note that while this definition for a Dynkin diagram may appear to be only a partial one because it says nothing about edges, it will become clear later that this definition is complete.

We now construct a LATKe with a single root and a single node in its Dynkin diagram corresponding to the cycle $\mathbf{P}^{2}$ in the blow-up of $\mathbf{C}^{3} / \mathbf{Z}_{3}$.

For the root space to be one-dimensional, the Cartan subalgebra must be two-dimensional. Let $\mathfrak{h}_{\mathfrak{L}}=\left\{H_{1}, H_{2}\right\}$ so that $\Lambda^{2} \mathfrak{h}_{\mathfrak{L}}$ is spanned by $H_{1} \wedge H_{2}$, and introduce candidates for one positive and one negative root vector, named $X$ and $Y$, respectively. All of the above lead to the following two equations:

$$
\begin{aligned}
& {\left[H_{1}, H_{2}, X\right]=\alpha\left(H_{1} \wedge H_{2}\right) X ;} \\
& {\left[H_{1}, H_{2}, Y\right]=-\alpha\left(H_{1} \wedge H_{2}\right) Y,}
\end{aligned}
$$

where $\alpha$ is the single root associated with $\Lambda^{2} \mathfrak{L}$. Normalizing $H_{1}$ and/or $H_{2}$ so that $\alpha\left(H_{1} \wedge H_{2}\right)=1$ (we should really be normalizing to $\alpha\left(H_{1} \wedge H_{2}\right)=-I_{111}$, where $I_{111}$ is the triple intersection number of the $\mathbf{P}^{2}$; however, the resulting algebra would be equivalent) gives

$$
\begin{aligned}
& {\left[H_{1}, H_{2}, X\right]=X ;} \\
& {\left[H_{1}, H_{2}, Y\right]=-Y .}
\end{aligned}
$$

All that is left is determining $\left[H_{1}, X, Y\right]$ and $\left[H_{2}, X, Y\right]$ such that the LATKe Jacobi identity would be satisfied. This identity requires, among other 
things, that $\left[H_{i}, X, Y\right], i=1,2$ are zero roots:

$$
\begin{aligned}
{\left[H_{1}, H_{2},\left[H_{i}, X, Y\right]\right]=} & {\left[\left[H_{1}, H_{2}, H_{i}\right], X, Y\right]+\left[H_{i},\left[H_{1}, H_{2}, X\right], Y\right] } \\
& +\left[H_{i}, X,\left[H_{1}, H_{2}, Y\right]\right] \\
= & 0+\left[H_{i}, X, Y\right]+\left[H_{i}, X,-Y\right]=0
\end{aligned}
$$

Therefore, $\left[H_{i}, X, Y\right] \in \mathfrak{h}_{\mathfrak{L}}$.

We now restrict the possibilities for $\left[H_{i}, X, Y\right]$ by limiting ourselves to simple LATKes, which we now define (recall that in the codimension 4 case, all the Lie algebras corresponding to orbifolds are simple). First we need the notion of ideals.

Definition 3.6 An ideal of $\mathfrak{L}$ is a subalgebra $\mathcal{I}$ that satisfies

$$
[\mathfrak{L}, \mathfrak{L}, \mathcal{I}] \subset \mathcal{I}
$$

Definition 3.7 A LATKe is simple if it is non-abelian and has no non-trivial ideals.

Requiring that our example would be simple means that $\left[H_{1}, X, Y\right]$ and $\left[H_{2}, X, Y\right]$ must be linearly independent, otherwise there would be a nontrivial ideal. It also requires that $\left[H_{i}, X, Y\right]$ and $H_{i}$ be linearly independent for each $i$.

So $\left[H_{i}, X, Y\right]$ are linearly independent combinations of $H_{1}$ and $H_{2}$. Any such linear combination can be transformed into another by linear redefinitions that do not affect the two commutators we already fixed in equation (3.16). Therefore, we may make a choice and we let

$$
\begin{aligned}
& {\left[H_{1}, X, Y\right]=H_{2}} \\
& {\left[H_{2}, X, Y\right]=H_{1} .}
\end{aligned}
$$

No more commutators need to be determined - all other commutators are defined by the requirement that the commutator map is trilinear and totally antisymmetric. One can easily verify that the LATKe Jacobi identity is satisfied and that there are no non-trivial ideals. Therefore, we have 
Example 3.1 The four-dimensional algebra spanned by $\left\{H_{1}, H_{2}, X, Y\right\}$ with the commutators

$$
\begin{aligned}
{\left[H_{1}, H_{2}, X\right] } & =X, \\
{\left[H_{1}, H_{2}, Y\right] } & =-Y, \\
{\left[H_{1}, X, Y\right] } & =H_{2}, \\
{\left[H_{2}, X, Y\right] } & =H_{1},
\end{aligned}
$$

is a simple LATKe. We name it $\mathfrak{L}_{3}$. Its Cartan subalgebra is 2-dimensional, its root space is 1-dimensional, and its Dynkin diagram consists of a single point:

\section{Uniqueness of the LATKe}

We have constructed the LATKe $\mathfrak{L}_{3}$ corresponding to the simplest codimension 6 orbifold singularity, $\mathbf{C}^{3} / \mathbf{Z}_{3}$. If the definition of LATKes has planted seeds for a generalization of the Du Val-Artin correspondence, then there should be a simple LATKe for each of the orbifolds $\mathbf{C}^{3} / \Gamma$, where $\Gamma$ is any discrete, finite subgroup of $S U(3)$ (these $\Gamma$ 's are classified in [17]).

Our goal now, therefore, is to classify all simple LATKes. In doing so, we would also learn more about their structure, which should help us in eventually constructing a physics theory appropriate to these algebras.

We begin by recalling that we can think of $\Lambda^{2} \mathfrak{h}_{\mathfrak{L}}$ as a set of operators acting on $\mathfrak{L}$, as in equation (3.12). More generally, we note that $\Lambda^{2} \mathfrak{L}$, not just $\Lambda^{2} \mathfrak{h}_{\mathfrak{L}}$, acts on $\mathfrak{L}$ as follows: if $X \wedge Y \in \Lambda^{2} \mathfrak{L}$ then its action on $Z \in \mathfrak{L}$ is given by

$$
X \wedge Y \quad: \quad Z \longmapsto[X, Y, Z]
$$

This action is called the adjoint action on $\mathfrak{L}$ and we denote it $\operatorname{ad}(X \wedge Y)$.

The LATKe Jacobi identity, equation (3.6), indicates that $\Lambda^{2} \mathfrak{L}$ acts as an inner derivation of $\mathfrak{L}$ : 
Definition 4.1 An operator $D$ on $\mathfrak{L}$ is a derivation of $\mathfrak{L}$ if it satisfies

$$
D[X, Y, Z]=[D X, Y, Z]+[X, D Y, Z]+[X, Y, D Z]
$$

The set of derivations of $\mathfrak{L}$ forms a Lie algebra with bracket given by

$$
\left[D_{1}, D_{2}\right]=D_{1} D_{2}-D_{2} D_{1}
$$

Definition 4.2 An inner derivation of $\mathfrak{L}$ is a derivation of $\mathfrak{L}$ which is a linear combination of operators of the form given in equation (4.1).

These two definitions are analogous to the ones given for conventional Lie algebras.

We shall denote the algebra of derivations of $\mathfrak{L}$ by $\operatorname{Der}(\mathfrak{L})$ and the inner derivations by $\operatorname{IDer}(\mathfrak{L})$.

Definition 4.3 For any LATKe $\mathfrak{L}$, the Lie algebra of $\mathfrak{L}$ is the space $\operatorname{IDer}(\mathfrak{L})$ with the commutator given by equation (4.3); we denote this Lie algebra $\mathfrak{g}_{\mathfrak{L}}$.

Note that the adjoint action defined before, given by

$$
\operatorname{ad}: X \wedge Y \in \Lambda^{2} \mathfrak{L} \longmapsto \operatorname{ad}(X \wedge Y) \in I \operatorname{Der}(\mathfrak{L})
$$

where

$$
\operatorname{ad}(X \wedge Y)(Z)=[X, Y, Z]
$$

satisfies

$$
[D, \operatorname{ad}(X \wedge Y)]=\operatorname{ad}(D(X \wedge Y)) \quad \forall D \in I \operatorname{Der}(\mathfrak{L})
$$

and is a surjective morphism of representations of $\operatorname{IDer}(\mathfrak{L})$.

It will be convenient to rewrite the LATKe Jacobi identity in the following form:

$$
\begin{aligned}
& {\left[X_{1}, X_{2},\left[X_{3}, X_{4}, X_{5}\right]\right]-\left[X_{3}, X_{4},\left[X_{1}, X_{2}, X_{5}\right]\right]=} \\
& =\left[\left[X_{1}, X_{2}, X_{3}\right], X_{4}, X_{5}\right]+\left[X_{3},\left[X_{1}, X_{2}, X_{4}\right], X_{5}\right]
\end{aligned}
$$


so that

$$
\left[X_{1} \wedge X_{2}, X_{3} \wedge X_{4}\right]=\left[X_{1}, X_{2}, X_{3}\right] \wedge X_{4}+X_{3} \wedge\left[X_{1}, X_{2}, X_{4}\right]
$$

or equivalently

$\left[\operatorname{ad}\left(X_{1} \wedge X_{2}\right), \operatorname{ad}\left(X_{3} \wedge X_{4}\right)\right]=\operatorname{ad}\left(\left[X_{1}, X_{2}, X_{3}\right] \wedge X_{4}\right)+\operatorname{ad}\left(X_{3} \wedge\left[X_{1}, X_{2}, X_{4}\right]\right)$

$\mathfrak{L}$ simple $\Longrightarrow \mathfrak{g}_{\mathfrak{L}}$ semi-simple We have shown that every LATKe $\mathfrak{L}$ has associated with it a Lie algebra $\mathfrak{g}_{\mathfrak{L}}$. We now prove a series of lemmas culminating in the result that if $\mathfrak{L}$ is a simple LATKe then its Lie algebra $\mathfrak{g}_{\mathfrak{L}}$ is semi-simple. Consequently, we will be able to utilize the well-known classification of semisimple Lie algebras as a tool for classifying the simple LATKes.

Lemma 4.1 If $\mathfrak{L}$ is simple then $\mathfrak{L}$ is irreducible as a representation of $\operatorname{IDer}(\mathfrak{L})$. Proof Let $W \subset \mathfrak{L}$ be an invariant subspace, i.e. IDer $(\mathfrak{L}): W \rightarrow W$. Then $[\mathfrak{L}, \mathfrak{L}, W] \subset W$ (see equations (4.4) and (4.5)). So $W$ is an ideal of $\mathfrak{L}$. Since $\mathfrak{L}$ is simple, $W=0$ or $\mathfrak{L}$.

Lemma 4.2 $\operatorname{IDer}(\mathfrak{L})$ is an ideal of $\operatorname{Der}(\mathfrak{L})$.

Proof Let $D \in \operatorname{Der}(\mathfrak{L})$ and let $X \wedge Y \in \Lambda^{2} \mathfrak{L}$ so $\operatorname{ad}(X \wedge Y) \in \operatorname{IDer}(\mathfrak{L})$. Then we have

$$
[D, \operatorname{ad}(X \wedge Y)] \cdot Z=D[X, Y, Z]-[X, Y, D Z]=[D X, Y, Z]+[X, D Y, Z]
$$

SO

$$
[D, \operatorname{ad}(X \wedge Y)]=\operatorname{ad}(D X \wedge Y)+\operatorname{ad}(X \wedge D Y)
$$

which is an inner derivation.

Fact 4.3 The space $\mathfrak{L}$ is a representation of $\operatorname{Der}(\mathfrak{L})$ and it is faithful by definition.

Lemma 4.4 If $\mathfrak{L}$ is simple then $\mathfrak{L}$ is irreducible as a representation of Der $(\mathfrak{L})$. Proof Any subspace $W \subset \mathfrak{L}$ that is invariant under $\operatorname{Der}(\mathfrak{L})$ is also invariant under its ideal IDer $(\mathfrak{L})$. By the proof of Lemma $4.1, W=0$ or $\mathfrak{L}$. 
Lemma 4.5 If $\mathfrak{L}$ is simple then $\operatorname{Der}(\mathfrak{L})$ is reductive.

Proof This follows from the fact that $\operatorname{Der}(\mathfrak{L})$ has a finite dimensional, faithful, irreducible representation, namely $\mathfrak{L}$.

Lemma 4.6 If $\mathfrak{L}$ is simple then any derivation of $\mathfrak{L}$ is an inner derivation, i.e. $\operatorname{Der}(\mathfrak{L})=\operatorname{IDer}(\mathfrak{L})$.

Proof Let $D$ be any derivation of $\mathfrak{L}$. Since $\operatorname{Der}(\mathfrak{L})$ is reductive, it has the form of a direct sum of commuting ideals. We already know that $\operatorname{IDer}(\mathfrak{L})$ is an ideal of $\operatorname{Der}(\mathfrak{L})$. Therefore, all we need to show is that if $D$ commutes with $\operatorname{IDer}(\mathfrak{L})$, then $D=0$. Assume

$$
[D, \operatorname{ad}(X \wedge Y)] \cdot Z=0 \quad \forall X, Y, Z \in \mathfrak{L}
$$

Expand this equation to

$$
D(\operatorname{ad}(X \wedge Y) \cdot Z)-\operatorname{ad}(X \wedge Y)(D \cdot Z)=D[X, Y, Z]-[X, Y, D Z]=0
$$

Repeating this for permutations of $X, Y$, and $Z$ and using the definition of derivations, we find that

$$
[D X, Y, Z]=[X, D Y, Z]=[X, Y, D Z]=0 \quad \forall X, Y, Z \in \mathfrak{L}
$$

Therefore, $D X$ is in the center of $\mathfrak{L}$ for any $X \in \mathfrak{L}$. But since $\mathfrak{L}$ is simple, it has no center. Therefore, $D X=0 \forall X$ so $D=0$.

Lemma 4.7 If $\mathfrak{L}$ is simple, then the center of IDer $(\mathfrak{L})$ is trivial.

Proof The argument in the proof of Lemma 4.6 shows that any derivation that commutes with $\operatorname{IDer}(\mathfrak{L})$ is zero.

Lemma 4.8 If $\mathfrak{L}$ is simple then $\operatorname{IDer}(\mathfrak{L})$ is semi-simple.

Proof Since IDer $(\mathfrak{L})$ is an ideal in a reductive Lie algebra, it is itself reductive. Any reductive Lie algebra is the direct sum of a semi-simple part and its center. Since IDer $(\mathfrak{L})$ has no center, it is semi-simple.

So we have shown that $\operatorname{IDer}(\mathfrak{L})=\mathfrak{g}_{\mathfrak{L}}$ is a semi-simple Lie algebra when $\mathfrak{L}$ is simple. We show next that there are very strong constraints on the roots 
and weights of $\mathfrak{g}_{\mathfrak{L}}$ that substantially limit the number of potential candidates for $\mathfrak{g}_{\mathfrak{L}}$.

Constraints on roots and weights of $\mathfrak{g}_{\mathfrak{L}}$ Since $\mathfrak{L}$ is a representation of $\mathfrak{g}_{\mathfrak{L}}$, so is $\Lambda^{2} \mathfrak{L}$. Recall from equations (4.4), (4.5), and (4.6) that we have a surjective morphism of representations,

$$
\operatorname{ad}: \Lambda^{2} \mathfrak{L} \longrightarrow \mathfrak{g}_{\mathfrak{L}}
$$

Therefore, there is a relation between the weights of $\Lambda^{2} \mathfrak{L}$ and the roots of $\mathfrak{g}_{\mathfrak{L}}$, which we shall now explore.

Let $\mathfrak{h}$ be the Cartan subalgebra of $\mathfrak{g}_{\mathfrak{L}}$, let $H \in \mathfrak{h}$, and let $X_{\beta_{i}} \in \mathfrak{L}$ be the weight vectors of $\mathfrak{L}$ with $\beta_{i}$ denoting weights of $\mathfrak{L}$ so that

$$
H\left(X_{\beta_{i}}\right)=\beta_{i}(H) X_{\beta_{i}}
$$

Then

$$
\begin{aligned}
{\left[H, \operatorname{ad}\left(X_{\beta_{i}} \wedge X_{\beta_{j}}\right)\right] } & =\operatorname{ad}\left(H\left(X_{\beta_{i}} \wedge X_{\beta_{j}}\right)\right) \\
& =\left(\beta_{i}(H)+\beta_{j}(H)\right) \operatorname{ad}\left(X_{\beta_{i}} \wedge X_{\beta_{j}}\right)
\end{aligned}
$$

It follows that any root of $\mathfrak{g}_{\mathfrak{L}}$ has the form $\beta_{i}+\beta_{j}$. However, since ad may have a non-trivial kernel, not all weights $\beta_{i}+\beta_{j}$ are necessarily roots.

Let the highest weight of $\mathfrak{L}$ as an irreducible representation of $\mathfrak{g}_{\mathfrak{L}}$ be denoted $\Lambda$. Now we prove a series of lemmas culminating in the result that $2 \Lambda-\alpha=$ $\Lambda+(\Lambda-\alpha)$ is a highest root of $\mathfrak{g}_{\mathfrak{L}}$, where $\alpha$ is a simple positive root of $\mathfrak{g}_{\mathfrak{L}}$. We do so by showing that there is a lowering operator $E_{-\alpha} \in \mathfrak{g}_{\mathfrak{L}}$ such that if $v_{\Lambda}$ is the highest weight vector of $\mathfrak{L}$, then $v_{\Lambda} \wedge\left(E_{-\alpha} v_{\Lambda}\right)$ is not in the kernel of ad.

The first step is to construct a (different) element in $\Lambda^{2} \mathfrak{L}$ which is not in the kernel of ad. Let $w_{0}$ be the element of the Weyl group of $\operatorname{IDer}(\mathfrak{L})$ that takes every positive root to a negative one and vice versa; then $w_{0}^{2}=1$.

Lemma 4.9 We have $\operatorname{ad}\left(v_{\Lambda} \wedge v_{w_{0} \Lambda}\right) \neq 0$ and $\operatorname{ad}\left(v_{\Lambda} \wedge v_{w_{0} \Lambda}\right) \in \mathfrak{h}$. 
Proof It is easy to see that $v_{\Lambda} \wedge v_{w_{0} \Lambda}$ generates the entire space $\Lambda^{2} \mathfrak{L}$. Therefore, its image under ad must be nonzero, otherwise ad itself would be identically zero, which would contradict that ad is onto $\operatorname{IDer}(\mathfrak{L})$.

Now, $\Lambda+w_{0} \Lambda$ is a root (see equation (4.17)). Since $w_{0}\left(\Lambda+w_{0} \Lambda\right)=w_{0} \Lambda+$ $w_{0}^{2} \Lambda=w_{o} \Lambda+\Lambda$ and $w_{0}$ takes positive roots to negative ones and vice versa, it follows that $\Lambda+w_{0} \Lambda=0$, or $w_{0} \Lambda=-\Lambda$. Therefore, $\operatorname{ad}\left(v_{\Lambda} \wedge v_{w_{0} \Lambda}\right) \in \mathfrak{h}$.

Lemma 4.10 In each simple ideal of IDer $(\mathfrak{L})$, there is a simple positive root $\alpha$ such that $\operatorname{ad}\left(v_{\Lambda} \wedge\left(E_{-\alpha} v_{\Lambda}\right)\right) \neq 0$.

Proof We have

$$
\begin{aligned}
\operatorname{ad}\left(v_{\Lambda} \wedge\left(E_{-\alpha} v_{\Lambda}\right)\right) \cdot v_{w_{0} \Lambda} & =\left[v_{\Lambda},\left(E_{-\alpha} v_{\Lambda}\right), v_{w_{0} \Lambda}\right] \\
& =\operatorname{ad}\left(\left(E_{-\alpha} v_{\Lambda}\right) \wedge v_{w_{0} \Lambda}\right) \cdot v_{\Lambda} \\
& =\operatorname{ad}\left(E_{-\alpha}\left(v_{\Lambda} \wedge v_{w_{0} \Lambda}\right)\right) \cdot v_{\Lambda} \\
& =\left[E_{-\alpha}, \operatorname{ad}\left(v_{\Lambda} \wedge v_{w_{0} \Lambda}\right)\right] \cdot v_{\Lambda} \\
& =\alpha\left(\operatorname{ad}\left(v_{\Lambda} \wedge v_{w_{o} \Lambda}\right)\right) E_{-\alpha} \cdot v_{\Lambda}
\end{aligned}
$$

If we now prove that for each simple ideal of $\operatorname{IDer}(\mathfrak{L})$, there is a simple root $\alpha$ such that $\alpha\left(\operatorname{ad}\left(v_{\Lambda} \wedge v_{w_{0} \Lambda}\right)\right) \neq 0$ and $E_{-\alpha} \cdot v_{\Lambda} \neq 0$, we will have proven the Lemma.

First, if we have $\alpha\left(\operatorname{ad}\left(v_{\Lambda} \wedge v_{w_{0} \Lambda}\right)\right) \neq 0$, then the identity

$$
\operatorname{ad}\left(\left(E_{-\alpha} v_{\Lambda}\right) \wedge v_{w_{0} \Lambda}\right)=\alpha\left(\operatorname{ad}\left(v_{\Lambda} \wedge v_{w_{0} \Lambda}\right)\right) E_{-\alpha}
$$

which can be deduced from equation (4.18), implies that $E_{-\alpha} v_{\Lambda} \neq 0$. So we need to show only that there is an $\alpha$ such that $\alpha\left(\operatorname{ad}\left(v_{\Lambda} \wedge v_{w_{0} \Lambda}\right)\right) \neq 0$.

Suppose first that $\operatorname{IDer}(\mathfrak{L})$ is simple. Then the root space is dual to $\mathfrak{h}$ so such an $\alpha$ automatically exists. Now suppose that $\operatorname{IDer}(\mathfrak{L})=\mathfrak{g}_{1} \oplus \cdots \oplus \mathfrak{g}_{k}$, where the $\mathfrak{g}_{i}$ are ideals in IDer( $\left.\mathfrak{L}\right)$. Then the Cartan subalgebra also has the form $\mathfrak{h}=\mathfrak{h}_{1} \oplus \cdots \oplus \mathfrak{h}_{k}$, and the root space decomposes to $\Delta=\Delta_{1} \oplus \cdots \oplus \Delta_{k}$ Now, suppose that $\alpha\left(\operatorname{ad}\left(v_{\Lambda} \wedge v_{w_{0} \Lambda}\right)\right)=0$ for all $\alpha \in \Delta_{1}$. Then $\operatorname{ad}\left(v_{\Lambda} \wedge v_{w_{0} \Lambda}\right) \in$ $\mathfrak{h}_{2} \oplus \cdots \oplus \mathfrak{h}_{k}$. Since $v_{\Lambda} \wedge v_{w_{0} \Lambda}$ generates all of $\Lambda^{2} \mathfrak{L}$, this means that the image of ad does not contain $\mathfrak{h}_{1}$, which contradicts the surjectivity of ad. 
Therefore, for each $i=1, \ldots, k$, there is a simple root $\alpha$ that satisfies both $\alpha\left(\operatorname{ad}\left(v_{\Lambda} \wedge v_{w_{0} \Lambda}\right)\right) \neq 0$, and $E_{-\alpha} \cdot v_{\Lambda} \neq 0$, so by equation $(4.18), v_{\Lambda} \wedge\left(E_{-\alpha} v_{\Lambda}\right)$ is not in the kernel of ad.

Note that no root of $\operatorname{IDer}(\mathfrak{L})$ is higher than $2 \Lambda-\alpha$ because there is no available pre-image under ad for such a root in $\Lambda^{2} \mathfrak{L}$, and ad is surjective. Putting everything together, we have

Lemma 4.11 The highest root $\theta$ of any simple component of $\mathfrak{g}_{\mathfrak{L}}$ is the sum of $\Lambda$, the highest weight of $\mathfrak{L}$, and a next-to-highest weight $\Lambda-\alpha$, where $\alpha$ is a simple positive root of a simple component of $\mathfrak{g}_{\mathfrak{L}}$ :

$$
\theta=\Lambda+(\Lambda-\alpha)
$$

or

$$
\theta+\alpha=2 \Lambda
$$

Only semi-simple Lie algebras which have a faithful irreducible representation $V$ whose highest weight $\Lambda$ satisfies condition (4.21) are potential candidates to be Lie algebras of some $\mathfrak{L}$. Our next step, therefore, is to find all semi-simple $\mathfrak{g}$ and highest weights $\Lambda$ satisfying this condition.

As it turns out, this same condition appeared in an entirely different context in Kac's work on Lie superalgebras [18], where all pairs of semi-simple Lie algebras $\mathfrak{g}$ and irreducible faithful representations $V$ with highest weight $\Lambda$ satisfying this condition are classified. The resulting list of pairs is the following.

If $\mathfrak{g}$ is not simple then $\mathfrak{g}=\mathfrak{s o}_{4} \simeq \mathfrak{s l}_{2} \times \mathfrak{s l}_{2}$ and $V$ is the standard fourdimensional (vector) representation.

If $\mathfrak{g}$ is simple then the following table constitutes the complete list of all 
pairs of $\mathfrak{g}$ and $V$ that satisfy the condition:

\begin{tabular}{llll}
$\mathfrak{g}$ & $\operatorname{dim} V$ & $\operatorname{dim} \mathfrak{g}$ & $\operatorname{dim} \Lambda^{2} V$ \\
\hline$A_{1}$ & 3 & 3 & 3 \\
$G_{2}$ & 7 & 14 & 21 \\
$A_{3}$ & 6 & 15 & 15 \\
$B_{3}$ & 8 & 21 & 28 \\
$B_{r \geq 2}$ & $2 r+1$ & $r(2 r+1)$ & $r(2 r+1)$ \\
$D_{r \geq 4}$ & $2 r$ & $r(2 r-1)$ & $r(2 r-1)$
\end{tabular}

The dimensions in the table uniquely identify each representation, except that for $D_{4}$ there are three different representations of dimension 8 , which are related to each other by the triality symmetry of $\mathfrak{s o}_{8}$.

Further conditions on $\mathfrak{g}_{\mathfrak{L}}$ and the construction of all $\mathfrak{L}$ The condition $2 \Lambda=\theta+\alpha$ is necessary, but not sufficient, to ensure that $\mathfrak{g}$ is the Lie algebra of some $\mathfrak{L}$. There is a further requirement: the intertwining map $\omega: \Lambda^{2} V \rightarrow \mathfrak{g}$ must yield a LATKe commutator in the following way. Let $v_{1}, v_{2}, v_{3} \in V$; then we define

$$
\left[v_{1}, v_{2}, v_{3}\right]=\left(\omega\left(v_{1} \wedge v_{2}\right)\right) \cdot v_{3}
$$

The expression on the right hand side must be antisymmetric in all three variables for it to define a LATKe commutator. Since it is already automatically antisymmetric in the first two variables, the only remaining requirement is

$$
\left(\omega\left(v_{1} \wedge v_{2}\right)\right) \cdot v_{3}=-\left(\omega\left(v_{3} \wedge v_{2}\right)\right) \cdot v_{1} \quad \forall v_{1}, v_{2}, v_{3} \in V
$$

which is equivalent to requiring

$$
\left.\omega\left(v_{1} \wedge v_{2}\right)\right) \cdot v_{1}=0 \quad \forall v_{1}, v_{2} \in V
$$

We first prove the following:

Lemma 4.12 None of the pairs of $\mathfrak{g}$ and $V$ in table 4.22 yields a LATKe commutator. 
Proof There are two steps to the proof. First, we construct explicitly the intertwining map $\omega: \Lambda^{2} V \rightarrow \mathfrak{g}$ for each pair of $\mathfrak{g}$ and $V$ in table 4.22. In each case, the adjoint representation, which is irreducible since $\mathfrak{g}$ is simple, appears exactly once in the decomposition of $\Lambda^{2} V$. Therefore, by Schur's lemma there is exactly one map $\omega$. Second, we show that $\omega$ does not satisfy equation (4.24) or (4.25), so it does not result in a LATKe commutator.

We begin with those pairs in table 4.22 that satisfy $\operatorname{dim} \mathfrak{g}=\operatorname{dim} \Lambda^{2} V$. We can construct the map $\omega$ for all such pairs simultaneously because all of them have the form $\mathfrak{s o}_{n}$ with $V$ the standard $n$-dimensional representation. That is obvious for $B_{r}$ and $D_{r}$, which stand for $\mathfrak{s o}_{2 r+1}$ and $\mathfrak{s o}_{2 r}$, respectively. For $A_{1}$ and $A_{3}$, recall that $A_{1}$ stands for $\mathfrak{s l}_{2} \simeq \mathfrak{s o}_{3}$ and $A_{3}$ stands for $\mathfrak{s l}_{4} \simeq \mathfrak{s o}_{6}$.

Let $\mathfrak{s o}_{n}$ be spanned by antisymmetric matrices $e_{a b}, a, b=1, \ldots, n, a<b$, such that

$$
\left\{e_{a b}\right\}_{\alpha \beta}=\delta_{a \alpha} \delta_{b \beta}-\delta_{a \beta} \delta_{b \alpha},
$$

i.e. $e_{a b}$ has +1 in the $a b$-th entry and -1 in the $b a$-th entry, with all other entries equal zero; if $a>b$ then $e_{a b}$ is defined by $e_{a b}=e_{b a}$. Let the standard representation $V$ be spanned by the standard basis $\left\{e_{a}\right\}, a=1, \ldots, n$, where

$$
\left(e_{a}\right)_{\alpha}=\delta_{a \alpha},
$$

i.e. $e_{a}$ has +1 in the $a$-th entry and zero elsewhere. It is straightforward to check that

$$
\begin{aligned}
& {\left[e_{a b}, e_{c d}\right]=\delta_{b c} e_{a d}+\delta_{a d} e_{b c}-\delta_{a c} e_{b d}-\delta_{b d} e_{a c}} \\
& e_{a b} \cdot\left(e_{c}\right)=\delta_{b c}\left(e_{a}\right)-\delta_{a c}\left(e_{b}\right)
\end{aligned}
$$

We want to construct $\omega: \Lambda^{2} V \rightarrow \mathfrak{g}$ which is intertwining, i.e.

$$
\begin{aligned}
{\left[X, \omega\left(v_{1} \wedge v_{2}\right)\right] } & =\omega\left(X\left(v_{1} \wedge v_{2}\right)\right) \\
& =\omega\left(\left(X \cdot v_{1}\right) \wedge v_{2}+v_{2} \wedge\left(X \cdot v_{2}\right)\right) \quad \forall X \in \mathfrak{g}, v_{i} \in V(4
\end{aligned}
$$

so we require

$$
\left[e_{a b}, \omega\left(e_{c} \wedge e_{d}\right)\right]=\omega\left(\left(e_{a b} \cdot e_{c}\right) \wedge e_{d}+e_{c} \wedge\left(e_{a b} \cdot e_{d}\right)\right) \quad \forall a, b, c, d .
$$


We now show that the map defined by

$$
\omega\left(e_{a} \wedge e_{b}\right)=e_{a b}
$$

satisfies this property.

Computing the right hand side of equation (4.31) for this map gives

$$
\begin{aligned}
& \omega\left(\left(e_{a b} \cdot e_{c}\right) \wedge e_{d}+e_{c} \wedge\left(e_{a b} \cdot e_{d}\right)\right)= \\
& =\omega\left(\left(\delta_{b c}\left(e_{a}\right)-\delta_{a c}\left(e_{b}\right)\right) \wedge e_{d}\right)+\omega\left(e_{c} \wedge\left(\delta_{b d}\left(e_{a}\right)-\delta_{a d}\left(e_{b}\right)\right)\right) \\
& =\delta_{b c} e_{a d}-\delta_{a c} e_{b d}+\delta_{b d} e_{c a}-\delta_{a d} e_{c b} .
\end{aligned}
$$

Comparing this with equation (4.28) proves that equation (4.32) is the desired map.

Now we check whether the condition (4.25) is satisfied. It is not:

$$
\omega\left(e_{a} \wedge e_{b}\right) \cdot e_{a}=e_{a b} \cdot e_{a}=-e_{b} \neq 0 .
$$

Therefore, no LATKe commutator arises from these $\mathfrak{s o}_{n}$ 's.

Now we construct the map $\omega$ for the pair $\mathfrak{g}_{2}$ with $\operatorname{dim} V=7$. As is wellknown, $\mathfrak{g}_{2}$ can be realized as the Lie algebra of derivations of the octonions, and the standard 7-dimensional representation of $\mathfrak{g}_{2}$ is given by its action on the (pure imaginary) octonions [19, 20].

Let $\left\{e_{i}\right\}, i=1, \ldots, 7$ be a basis for the pure imaginary octonions, and for our representation $V$. Define the map $\omega: \Lambda^{2} V \rightarrow \mathfrak{g}_{2}$ by

$$
\omega\left(e_{i} \wedge e_{j}\right) \cdot e_{k}=\left[\left[e_{i}, e_{j}\right], e_{k}\right]-3\left(\left(e_{i} e_{j}\right) e_{k}-e_{i}\left(e_{j} e_{k}\right)\right),
$$

where $[x, y]=x y-y x$, with the multiplication being the one in the octonion algebra, given for example by the standard Fano plane [19, 20]. (Recall that the octonions are not associative, so the second term above does not in general vanish; also, this second term is antisymmetric under $e_{i} \leftrightarrow e_{j}$, as is the first term). It can be shown [21] that every $\omega\left(e_{i} \wedge e_{j}\right)$ is a derivation of the octonions, and that every derivation of the octonions is a linear combination of derivations of the form $\omega\left(e_{i} \wedge e_{j}\right)$. Therefore, the map $\omega$ of Equation (4.35) is onto $\mathfrak{g}_{2}$. 
Also, it is straightforward to see that $\omega$ is intertwining. Since $\mathfrak{g}_{2}$ appears exactly once in the decomposition $\Lambda^{2} V=\mathfrak{g}_{2} \oplus V$, the map $\omega$ is unique.

As before, we now check whether the condition (4.25) is satisfied. It is enough to show it is not satisfied in one case:

$$
\omega\left(e_{1} \wedge e_{2}\right) \cdot e_{1}=4 e_{2} \neq 0 .
$$

Therefore, no LATKe commutator arises from $\mathfrak{g}_{2}$.

We have now but one more pair to check: $B_{3}=\mathfrak{s o}_{7}$ with $\operatorname{dim} V=8$, where $V$ is the spin representation. The representation $\Lambda^{2} V$ decomposes into $\mathfrak{s o}_{7} \oplus V_{7}$, where $\mathfrak{s o}_{7}$ stands for the adjoint representation and $V_{7}$ is the standard seven-dimensional representation of $\mathfrak{s o}_{7}$. Since $\mathfrak{s o}_{7}$ appears once in the decomposition, there is a unique intertwining map $\omega: \Lambda^{2} V \rightarrow \mathfrak{s o}_{7}$. We now explicitly construct this map and show that it does not yield a LATKe commutator.

We first construct the spin representation of $\mathfrak{s o}_{7}$ explicitly, following [20]. Let $C\left(V_{7}, Q\right)$ be the Clifford algebra generated by $V_{7}$ with the quadratic form

$$
Q=\left(\begin{array}{ccc}
0 & I_{3} & 0 \\
I_{3} & 0 & 0 \\
0 & 0 & 1
\end{array}\right),
$$

where $I_{3}$ is the $3 \times 3$ identity matrix. Then $\mathfrak{s o}_{7}$ is a Lie subalgebra of $C\left(V_{7}, Q\right)$ via the embedding

$$
\psi \cdot \phi^{-1}: \mathfrak{s o}_{7} \rightarrow C\left(V_{7}, Q\right),
$$

where $\phi: \Lambda^{2} V_{7} \rightarrow \mathfrak{s o}_{7}$ is an isomorphism given by

$$
\phi_{a \wedge b}(v)=2(Q(b, v) a-Q(a, v) b), \quad v \in V_{7},
$$

and $\psi: \Lambda^{2} V_{7} \rightarrow C\left(V_{7}, Q\right)$ is an embedding given by

$$
\psi: a \wedge b \mapsto a \cdot b-Q(a, b)
$$

Decompose $V_{7}$ into $W \oplus W^{\prime} \oplus U$, where $W$ and $W^{\prime}$ are three-dimensional isotropic subspaces and $U$ is a one dimensional subspace orthogonal to them. 
Then there is an action of $C\left(V_{7}, Q\right)$ on $\Lambda^{\bullet} W=\sum_{i=0}^{3} \Lambda^{i} W$, whose restriction to $\mathfrak{s o}_{7}$ will be the spin representation. It is given as follows.

Let $\zeta \in \Lambda^{\bullet} W$, and let $l: V_{7} \rightarrow \operatorname{End}\left(\Lambda^{\bullet} W\right)$ be given by:

$$
\begin{aligned}
& l(w) \cdot \zeta=w \wedge \zeta \quad w \in W, \\
& l\left(w^{\prime}\right) \cdot \zeta=D_{w^{\prime}}(\zeta), \quad w^{\prime} \in W^{\prime}, \\
& l\left(e_{0}\right) \cdot \zeta=\left\{\begin{array}{cc}
\zeta & \zeta \in \Lambda^{\text {even }} W \\
-\zeta & \zeta \in \Lambda^{\text {odd } W}
\end{array}, \quad e_{0} \in U, Q\left(e_{0}, e_{0}\right)=1 .\right.
\end{aligned}
$$

where

$$
\begin{aligned}
& D_{w^{\prime}}(1)=0 \\
& D_{w^{\prime}}(w)=2 Q\left(w, w^{\prime}\right), \\
& D_{w^{\prime}}\left(w_{1} \wedge w_{2}\right)=-\phi_{w_{1} \wedge w_{2}}\left(w^{\prime}\right), \\
& D_{w^{\prime}}\left(w_{1} \wedge w_{2} \wedge w_{3}\right)=D_{w^{\prime}}\left(w_{1} \wedge w_{2}\right) \wedge w_{3}+D_{w^{\prime}}\left(w_{3}\right) w_{1} \wedge w_{2} .
\end{aligned}
$$

The map $l$ gives the action of $V_{7}$ on $\Lambda^{\bullet} W$ from which the action of $C\left(V_{7}, Q\right)$ on $\Lambda^{\bullet} W$ can be deduced. This action restricted to $\mathfrak{s o}_{7}$ is the spin representation.

The action of $\mathfrak{s o}_{7}$ on $\Lambda^{2}\left(\Lambda^{\bullet} W\right)$ is then given by

$$
X\left(\zeta_{1} \wedge \zeta_{2}\right)=\left(l(X) \cdot \zeta_{1}\right) \wedge \zeta_{2}+\zeta_{1} \wedge\left(l(X) \cdot \zeta_{2}\right), \quad X \in \mathfrak{s o}_{7}
$$

Now we turn to constructing the map $\omega$ satisfying the intertwining condition, i.e.

$$
[X, \omega(\eta)]=\omega(X(\eta)) \quad \forall X \in \mathfrak{s o}_{7}, \eta \in \Lambda^{2}\left(\Lambda^{\bullet} W\right) .
$$

If $\eta \in \Lambda^{2}\left(\Lambda^{\bullet} W\right)$ is a unique (up to scalar) element satisfying

$$
H(\eta)=\alpha(H) \eta \quad \forall H \in \mathfrak{h} \subset \mathfrak{s o}_{7},
$$

where $\mathfrak{h}$ is the Cartan subalgebra of $\mathfrak{s o}_{7}$ and $\alpha \in \mathfrak{h}^{*}$ is a root, then since $\omega$ is onto $\mathfrak{s o}_{7}$ and is intertwining, $\omega(\eta)$ must be a non-zero multiple of the $\alpha$ root vector in $\mathfrak{s o}_{7}$. 
Let $\left\{e_{i}\right\}, i=1,2,3$ denote a basis for $W$, let $\left\{e_{i+3}\right\}, i=1,2,3$ denote a basis for $W^{\prime}$, and let $e_{0}$ be a basis for $U$. With the notation given in Appendix $\mathrm{C}$, let $H_{1}=\mu_{11}, H_{2}=\mu_{22}$, and $H_{3}=\mu_{33}$ form the Cartan subalgebra. Consider the element $1 \wedge e_{1} \in \Lambda^{2}\left(\Lambda^{\bullet} W\right)$. Then

$$
H_{1}\left(1 \wedge e_{1}\right)=0 ; \quad H_{2}\left(1 \wedge e_{1}\right)=-1 \wedge e_{1} ; \quad H_{3}\left(1 \wedge e_{1}\right)=-1 \wedge e_{1} .
$$

One can check that $1 \wedge e_{1}$ is the only element in $\Lambda^{2}\left(\Lambda^{\bullet} W\right)$ (up to scalar) with these eigenvalues. In $\mathfrak{s o}_{7}$, the unique element (up to scalar) with these eigenvalues is $\rho_{23}$ (see Appendix C). Therefore, we may set

$$
\omega\left(1 \wedge e_{1}\right)=\rho_{23}
$$

The rest of $\omega$ is fully determined by repeated applications of the intertwining condition and the action of $\mathfrak{s o}_{7}$ on $\Lambda^{2}\left(\Lambda^{\bullet} W\right)$. We provide the resulting $\omega$ in Appendix C.

It is now straightforward to check that the map $\omega$ does not satisfy Equation (4.24):

$$
\omega\left(f_{1} \wedge f_{5}\right) \cdot f_{4}=-2 f_{1} \neq-\omega\left(f_{1} \wedge f_{4}\right) \cdot f_{5}=f_{1}
$$

where $\left\{f_{k}\right\}, k=1, \ldots, 8$ is the basis for $\Lambda^{\bullet} W$ given in Appendix C.

Therefore, we do not get a LATKe from any of the simple Lie algebras in table 4.22 , and the proof of the lemma is complete.

We are now left with only one candidate: $\mathfrak{s o}_{4}$ with its standard representation. Recall that $\mathfrak{s o}_{4}$ is not a simple Lie algebra but has two simple factors, $\mathfrak{s o}_{4} \simeq \mathfrak{s l}_{2} \times \mathfrak{s l}_{2}$. So its adjoint representation is not irreducible, Schur's lemma does not apply, and the intertwining map $\omega$ constructed above for $\mathfrak{s o}_{n}$ is not unique for this case. We can construct another one.

We will show that the other map does lead to a LATKe commutator and in fact yields the LATKe $\mathfrak{L}_{3}$ which we constructed in Section 3.

Using the same notation as before, the basis for $\mathfrak{s o}_{4}$ is

$$
\left\{e_{12}, e_{13}, e_{14}, e_{23}, e_{24}, e_{34}\right\}
$$


and the basis for $V$ is

$$
\left\{e_{1}, e_{2}, e_{3}, e_{4}\right\} .
$$

We define $\phi: \Lambda^{2} V \rightarrow \mathfrak{g}$ explicitly by

$$
\begin{aligned}
& \phi\left(e_{1} \wedge e_{2}\right)=e_{34}, \\
& \phi\left(e_{1} \wedge e_{3}\right)=-e_{24}, \\
& \phi\left(e_{1} \wedge e_{4}\right)=e_{23}, \\
& \phi\left(e_{2} \wedge e_{3}\right)=e_{14}, \\
& \phi\left(e_{2} \wedge e_{4}\right)=-e_{13}, \\
& \phi\left(e_{3} \wedge e_{4}\right)=e_{12} .
\end{aligned}
$$

It is straightforward to check that this map satisfies the intertwining condition, equation (4.31).

We now use the map $\phi$ to construct the LATKe commutator. The dimension of the candidate for $\mathfrak{L}$ is $\operatorname{dim} V=4$, so there are only four commutators to calculate:

$$
\begin{aligned}
& {\left[e_{1}, e_{2}, e_{3}\right]=\phi\left(e_{1} \wedge e_{2}\right) \cdot e_{3}=e_{34} \cdot e_{3}=-e_{4} ;} \\
& {\left[e_{1}, e_{2}, e_{4}\right]=\phi\left(e_{1} \wedge e_{2}\right) \cdot e_{4}=e_{34} \cdot e_{4}=e_{3} ;} \\
& {\left[e_{1}, e_{3}, e_{4}\right]=\phi\left(e_{1} \wedge e_{3}\right) \cdot e_{4}=-e_{24} \cdot e_{4}=-e_{2} ;} \\
& {\left[e_{2}, e_{3}, e_{4}\right]=\phi\left(e_{2} \wedge e_{3}\right) \cdot e_{4}=e_{14} \cdot e_{4}=e_{1} .}
\end{aligned}
$$

With the following change of variables

$$
X=\frac{1}{\sqrt{2}}\left(e_{1}+i e_{4}\right) ; Y=\frac{1}{\sqrt{2}}\left(e_{1}-i e_{4}\right) ; H_{1}=-i e_{2} ; H_{2}=e_{3},
$$

we see that this algebra is precisely $\mathfrak{L}_{3}$ as given in equations (3.20).

This construction of $\mathfrak{L}_{3}$ generalizes to a LAnKe $\mathfrak{L}_{n}$, as shown in Appendix B.

We summarize the results of this section in one theorem:

Theorem 4.1 There is precisely one simple LATKe, namely $\mathfrak{L}_{3}$; it is four dimensional, it corresponds to the singularity $\mathbf{C}^{3} / \mathbf{Z}_{3}$, its Lie algebra $\mathfrak{g}_{\mathfrak{L}_{3}}$ is 
$\mathfrak{s o}_{4} \simeq \mathfrak{s l}_{2} \times \mathfrak{s l}_{2}$, and its Dynkin diagram consists of one node. Its commutators are given by equation (3.20) or (4.52).

We comment that the theorem is equivalent to the following statement. Let $V$ be a vector space with a non-degenerate symmetric bilinear form so that $\Lambda^{2} V \simeq \mathfrak{s o}(V)$. There is a natural action of $\Lambda^{2} V$ on $V$ which induces a map $\omega_{3}: V \otimes V \otimes V \rightarrow V$, antisymmetric in the first two factors. Let $\bar{\omega}_{3}: \Lambda^{3} V \rightarrow V$ be the fully-antisymmetric version of $\omega_{3}$. The theorem says that, if we assume that the action of $\mathfrak{s o}(V)$ on $V$ was irreducible, then there is a unique vector space $V$ for which $\bar{\omega}_{3}$ is both non-trivial and satisfies the Jacobi identity of the third kind (equation (3.6)). That vector space is four dimensional and $\Lambda^{2} V$ consists of skew-symmetric 4-matrices.

\section{The Physics of LATKes}

We have now reached an important juncture. Having found the unique, simple LATKe, we ask ourselves: are there any applications of the LATKe to physics?

The first type of physical theory that comes to mind in applying Lie algebras to physics is gauge theory. Can we generalize gauge theory for LATKes?

The answer is "yes," and we do so in the context of particle physics.

\subsection{LATKe representations and LATKe groups}

Representations Whenever Lie algebras are applied to particle physics, particles are viewed as basis vectors of representations of the Lie algebra. For example, gauge fields form the adjoint representation of the gauge group; quarks form the three-dimensional representation of color- $S U(3)$; electrons form the two-dimensional spin representation of $S U(2)$ [22]. Therefore, in order to apply LATKes to particle physics, we must define a "representation" for LATKes. 
To do so, we review the standard Lie algebra case. A representation of a Lie algebra $\mathfrak{g}$ is a map from $\mathfrak{g}$ to operators on some vector space $V$ :

$$
\rho: \mathfrak{g} \longrightarrow \operatorname{End}(V)
$$

and it respects the commutator in the following way:

$$
[\rho(X), \rho(Y)]=\rho([X, Y])
$$

A particular representation that utilizes the commutator in a natural way is the adjoint representation, given by the following map:

$$
\begin{aligned}
\text { ad } & : \quad \mathfrak{g} \longrightarrow \operatorname{End}(\mathfrak{g}) \\
\operatorname{ad}(X) & : \quad Y \longmapsto[X, Y] .
\end{aligned}
$$

This map satisfies the condition

$$
[\operatorname{ad}(X), \operatorname{ad}(Y)]=\operatorname{ad}([X, Y])
$$

which is equivalent to the standard Jacobi identity. The condition in equation (5.2) is a generalization of the relation given in equation (5.5) and it reduces to it when $\rho=$ ad.

Now, we define a representation for a LATKe. We begin by defining the analog of the adjoint representation: it is also a map that utilizes the commutator in a natural way, and we have in fact seen it before (equation (4.1)):

$$
\begin{aligned}
& \text { ad }: \quad \mathfrak{L} \wedge \mathfrak{L} \longrightarrow \operatorname{End}(\mathfrak{L}) \\
& \operatorname{ad}(X \wedge Y) \quad: \quad Z \longmapsto[X, Y, Z] .
\end{aligned}
$$

The map ad satisfies the condition

$$
\left[\operatorname{ad}\left(X_{1} \wedge X_{2}\right), \operatorname{ad}\left(X_{3} \wedge X_{4}\right)\right]=\operatorname{ad}\left(\left[X_{1}, X_{2}, X_{3}\right] \wedge X_{4}\right)+\operatorname{ad}\left(X_{3} \wedge\left[X_{1}, X_{2}, X_{4}\right]\right)
$$

which is equivalent to the LATKe Jacobi identity.

If we generalize equations (5.6) and (5.8), we have 
Definition 5.1 A representation of a LATKe $\mathfrak{L}$ is a map

$$
\rho: \Lambda^{2} \mathfrak{L} \longrightarrow \operatorname{End}(V)
$$

for some vector space $V$ subject to the condition

$$
\left[\rho\left(X_{1} \wedge X_{2}\right), \rho\left(X_{3} \wedge X_{4}\right)\right]=\rho\left(\left[X_{1}, X_{2}, X_{3}\right] \wedge X_{4}\right)+\rho\left(X_{3} \wedge\left[X_{1}, X_{2}, X_{4}\right]\right)
$$

This condition is analogous to equation (5.2) and it generalizes the LATKe Jacobi identity (see equations (3.6), (4.7) and (4.8)).

Groups Another fundamental ingredient whenever Lie algebras are applied to particle physics is the Lie group, which plays the role of a symmetry of the physical system.

Therefore, in order to apply LATKes to particle physics, we also should define a "Lie group of the third kind," or a "LATKe group," which would be related to the LATKe in a way analogous to the relation between an ordinary Lie group and its Lie algebra. The LATKe group could then play the role of some kind of generalized symmetry in the yet-to-be constructed physics of LATKes.

Here, we run into trouble: we have found it impossible to generalize the notion of a Lie group to something we might have called a LATKe group. While there does happen to be a Lie group associated with the LATKe, namely the exponential of $\mathfrak{g}_{\mathfrak{L}}$, it is not in any way an exponential of the LATKe itself. So it is not a "LATKe group."

Since we have no LATKe analog of a Lie group, it would be impossible to generalize in a natural way any application of Lie algebras to physics in which the Lie group is an indispensable ingredient. We are limited to applications in which the only necessary mathematical ingredients are those for which we do have a LATKe analog.

Since we wish to generalize gauge theory for LATKes, we must check whether the Lie group itself, which plays the role of the gauge group, is an indispensable ingredient in the construction of gauge theory. If it is, we would 
be unable to generalize it for LATKes. In the next section we demonstrate that the gauge group is not indispensable in gauge theory by rephrasing the original theory of Yang and Mills [23] so that all group tranformations are rewritten as Lie algebra actions. That sets the stage for a natural generalization of Yang-Mills theory to LATKes, which we construct in Section 5.3.

\subsection{Traditional Yang-Mills theory}

Let $\psi$ be a wave function describing a field in some representation $\rho$ of a Lie algebra $\mathfrak{g}$. Let $\mathfrak{g}$ be spanned by basis elements $T^{i}$ with $i=1, \ldots, \operatorname{dim} \mathfrak{g}$ (these are analogues of Pauli spin matrices in the $\mathfrak{s u}(2)$ case). A gauge transformation acts via

$$
\delta \psi=-2 i \Theta^{i} T^{i} \psi
$$

where each $\Theta^{i}, i=1, \ldots, \operatorname{dim} \mathfrak{g}$ is a space-time dependent field, and each $T^{i}$ acts on $\psi$ via the representation $\rho$.

Invariance under such transformations is preserved only if we require derivatives of $\psi$ to appear in the combination

$$
\left(\partial_{\mu}-i g B_{\mu}\right) \psi
$$

where $g$ is a coupling constant and

$$
B_{\mu}=2 b_{\mu}^{j} T^{j}
$$

with $b_{\mu}^{j}$ space-time dependent. The combination in equation (5.12) is the wellknown covariant derivative, and $B_{\mu}$ is the well-known gauge field. Under the gauge transformation, $B_{\mu}$ transforms via

$$
\delta B_{\mu}=2 i\left[B_{\mu}, \Theta^{i} T^{i}\right]-\frac{2}{g}\left(\partial_{\mu} \Theta^{i}\right) T^{i} .
$$

With these transformations, we have

$$
\delta\left[\left(\partial_{\mu}-i g B_{\mu}\right) \psi\right]=-2 i \Theta^{i} T^{i}\left(\left(\partial_{\mu}-i g B_{\mu}\right) \psi\right),
$$

as would be expected from equation (5.11). 
We define the field strength $F_{\mu \nu}$ by

$$
\begin{aligned}
F_{\mu \nu} & =\partial_{\nu} B_{\mu}-\partial_{\mu} B_{\nu}+i g\left[B_{\mu}, B_{\nu}\right] \\
& =2 f_{\mu \nu}^{i} T^{i}
\end{aligned}
$$

where $f_{\mu \nu}^{i}$ are space-time dependent and the commutator $\left[B_{\mu}, B_{\nu}\right]$ is the one defining the Lie algebra $\mathfrak{g}$. Under the gauge transformation, $F_{\mu \nu}$ transforms by

$$
\delta F_{\mu \nu}=2 i\left[F_{\mu \nu}, \Theta^{i} T^{i}\right] .
$$

Now we have all the necessary ingredients to write the Lagrangian:

$$
L=-\frac{1}{4} f_{\mu \nu} f_{\mu \nu}-\bar{\psi} \gamma_{\mu}\left(\partial_{\mu}-2 i g b_{\mu}^{i} T^{i}\right) \psi-m \psi \bar{\psi} .
$$

The Lagrangian is invariant under gauge transformations. From this Lagrangian, the equations of motion of the gauge theory are derived.

\subsection{LATKe Yang-Mills, or pure Yang-Mills with matter}

We can now generalize gauge theory by replacing Lie algebras with LATKes and replacing representations of Lie algebras with representations of LATKes.

That means we now let $\psi$ be a field in a representation $\rho$ of a LATKe as defined in equation (5.9), and we let $\Theta_{a b}\left(e_{a} \wedge e_{b}\right) \in \Lambda^{2} \mathfrak{L}$ act on $\psi$ via that representation in lieu of the action of $\Theta^{i} T^{i}$ of the standard case of Section 5.2. Here, $\Theta_{a b}, a, b=1, \ldots, \operatorname{dim} \mathfrak{L}$ are space-time dependent fields antisymmetric in $a$ and $b$. We replace every occurrence of $\Theta^{i} T^{i}$ in Section 5.2 by $\Theta_{a b}\left(e_{a} \wedge e_{b}\right)$, and every index $\{i\}$ by an antisymmetric double-index $\{a b\}$.

If we now inspect the resulting equations - which are the equations of LATKe Yang-Mills theory - we find that the LATKe $\mathfrak{L}$ appears only through $\Lambda^{2} \mathfrak{L}$. This is so because we were using representations of the LATKe in the construction, and those involve $\Lambda^{2} \mathfrak{L}$ rather than $\mathfrak{L}$ (see Definition 5.1).

We may now note that for $\mathfrak{L}=\mathfrak{L}_{3}, \Lambda^{2} \mathfrak{L}$ is isomorphic to $\mathfrak{g}_{\mathfrak{L}}$ (the map $\omega$ is an isomorphism in this case) so that $\Lambda^{2} \mathfrak{L}$ is in fact the Lie algebra $\mathfrak{g}_{\mathfrak{L}}$. 
And, we also observe that the way in which $\Lambda^{2} \mathfrak{L}$ appears in our LATKe YangMills theory is precisely the same as the way ordinary Lie algebras appear in traditional Yang-Mills theory, i.e. exactly as in Section 5.2. It turns out that the definition of representations of a LATKe has conspired with the structure of the Lie algebra $\mathfrak{g}_{\mathfrak{L}}$ of the LATKe to turn LATKe Yang-Mills theory into a conventional Yang-Mills theory with Lie algebra $\mathfrak{g}_{\mathfrak{s}}$ ! And, now it is inevitable that we would think of LATKe Yang-Mills theory as a conventional Yang-Mills theory with gauge group $\exp \left(\mathfrak{g}_{\mathfrak{L}}\right)$.

Yet, there is an essential and crucial difference between conventional YangMills and LATKe Yang-Mills: in conventional Yang Mills theory, we have what is known as "pure Yang-Mills theory," where the gauge fields $B_{\mu}$, which live in the adjoint representation of the gauge group, are the only fields. There are no matter fields - that is, no field $\psi$ appears - and the Lagrangian consists only of the first term of equation (5.18). In general, for physical theories to include matter fields they typically have to be put in by hand.

But in the LATKe Yang-Mills theory, this is not the case. Built into the theory is not just the adjoint representation $\Lambda^{2} \mathfrak{L}$ of $\mathfrak{g}_{\mathfrak{L}}$, but also the adjoint representation of the LATKe itself, i.e. $\mathfrak{L}$. This representation is in fact a matter representation of $\mathfrak{g}_{\mathfrak{L}}$ and an inseparable part of pure LATKe YangMills theory.

Therefore, unlike pure Yang-Mills theory, pure LATKe Yang-Mills theory automatically includes matter, without the need to put it in by hand.

\subsection{Gauge theory for $\mathfrak{L}_{3}$}

For $\mathfrak{L}=\mathfrak{L}_{3}$, we have $\mathfrak{g}_{\mathfrak{L}}=\mathfrak{s o}_{4} \simeq \mathfrak{s l}_{2} \times \mathfrak{s l}_{2}$ and $\mathfrak{L}$ forms the $(2,2)$ representation (see Theorem 4.1). The unitary version of $\exp \left(\mathfrak{g}_{\mathfrak{L}}\right)$ is $S O(4)$ or $S U(2) \times S U(2)$. The pure LATKe Yang-Mills theory for $\mathfrak{L}_{3}$ is therefore an $S O(4)$ or $S U(2) \times$ $S U(2)$ gauge theory with matter in the $(2,2)$ representation. 


\subsection{LATKe Yang-Mills theory from $G_{2}$ manifolds}

Here we show that $\mathbf{C}^{3} / \mathbf{Z}_{3}$, which is the singularity corresponding to the LATKe $\mathfrak{L}_{3}$, indeed arises in a $G_{2}$ space, as we encountered in [7].

Let $X$ be the manifold of $G_{2}$ holonomy which is asymptotic at infinity to a cone over $Y=\mathbf{S}^{3} \times \mathbf{S}^{3}=S U(2)^{3} / S U(2)_{\Delta}$, where the equivalence relation $\Delta$ is $\left(g_{1}, g_{2}, g_{3}\right) \sim\left(g_{1} h, g_{2} h, g_{3} h\right), g_{i}, h \in S U(2)[24,25,5,7]$.

Let $\gamma \in \mathbf{Z}_{3}$ act on $Y$ as follows:

$$
\gamma:\left(g_{1}, g_{2}, g_{3}\right) \longmapsto\left(\gamma g_{1}, \gamma g_{2}, g_{3}\right) ; \gamma=\left(\begin{array}{cc}
e^{2 \pi i / 3} & 0 \\
0 & e^{-2 \pi i / 3}
\end{array}\right) .
$$

The metric of $X$ is preserved under this action $[5,7]$. Using the equivalence relation $\Delta$ to set $g_{1}=1$, we rewrite this action as

$$
\left(1, g_{2}, g_{3}\right) \longmapsto\left(1, \gamma g_{2} \gamma^{-1}, g_{3} \gamma^{-1}\right) .
$$

We can obtain $X$ from $Y$ by filling in one of the $S U(2)$ factors to a ball that includes the origin (recall that $S U(2) \sim \mathbf{S}^{3}$ ). Let us fill in the third $S U(2)$ factor, and study the singularity at the origin.

We may write

$$
g_{2}=\left(\begin{array}{cc}
z_{1} & z_{2} \\
-\bar{z}_{2} & \bar{z}_{1}
\end{array}\right), \quad\left|z_{1}^{2}\right|+\left|z_{2}^{2}\right|=1 ; \quad g_{3}=\left(\begin{array}{cc}
w_{1} & -\bar{w}_{2} \\
w_{2} & \bar{w}_{1}
\end{array}\right),
$$

where $z_{i}$ and $w_{i}$ are complex variables. Then the action of $\gamma$ becomes

$$
\gamma:\left(z_{1}, z_{2}, w_{1}, w_{2}\right) \longmapsto\left(z_{1}, e^{4 \pi i / 3} z_{2}, e^{4 \pi i / 3} w_{1}, e^{4 \pi i / 3} w_{2}\right) .
$$

This action is singular at $z_{2}=w_{1}=w_{2}=0$. The locus of the singularity is the circle $z_{1}=e^{i \theta}$.

The singularity itself is equivalent to the one which we used in Section 3 to construct $\mathfrak{L}_{3}$, with the $\mathbf{C}^{3}$ given by coordinates $\left\{z_{2}, w_{1}, w_{2}\right\}$ and $\epsilon=e^{4 \pi i / 3}$ (see equation (3.9)).

Therefore, we argue that the theory obtained from M-theory compactified on a $G_{2}$ space with a circle of $\mathbf{C}^{3} / \mathbf{Z}_{3}$ singularities is governed by the LATKe 
Yang-Mills theory we constructed in Section 5.3, i.e. it is an $S U(2) \times S U(2)$ or $S O(4)$ gauge theory on $M^{4} \times \mathbf{S}^{1}$ with one matter field in the $(2,2)$ representation of $S U(2) \times S U(2)$, which is the vector representation of $S O(4)$. Since this compactification is supersymmetric (it has $G_{2}$ holonomy), the LATKe Yang-Mills is also supersymmetric $(\mathcal{N}=1)$.

Similarly, we argue that the same physics would result if a $\mathbf{C}^{3} / \mathbf{Z}_{3}$ singularity appears in a Calabi-Yau space on which a string theory is compactified.

\section{$5.6 \quad$ Unbreakability of $\mathfrak{g}_{\mathfrak{L}}$}

We have shown that $\mathfrak{L}_{3}$ leads to an $\mathfrak{s u}(2) \times \mathfrak{s u}(2)$ gauge theory with matter. A physicist conditioned to search for the standard model is immediately led to the following question: can we break $\mathfrak{s u}(2) \times \mathfrak{s u}(2)$ to $\mathfrak{s u}(2) \times \mathfrak{u}(1)$, which is the gauge symmetry of electroweak theory and part of the standard model?

Recall that the commutators of $\mathfrak{L}_{3}$ were determined from the map $\phi$ : $\Lambda^{2} V \rightarrow \mathfrak{g}$, defined in equation (4.51), which was required to satisfy the antisymmetry condition in equation (4.24) or (4.25). Now we show that if $\mathfrak{s u}(2) \times \mathfrak{s u}(2)$ is broken to $\mathfrak{s u}(2) \times \mathfrak{u}(1)$, none of the commutators of $\mathfrak{L}_{3}$ are well-defined; in other words, they disappear.

Let $\left\{u_{1}, \ldots, u_{6}\right\}$ be a basis for $\mathfrak{s o}(4)$ for which the $\mathfrak{s u}(2) \times \mathfrak{s u}(2)$ structure is explicit:

$$
\begin{array}{lll}
u_{1}=e_{12}+e_{34}, & u_{2}=-e_{13}+e_{24}, & u_{3}=e_{14}+e_{23}, \\
u_{4}=e_{12}-e_{34}, & u_{5}=e_{13}+e_{24}, & u_{6}=e_{14}-e_{23} .
\end{array}
$$

Here, $\left\{u_{1}, u_{2}, u_{3}\right\}$ span one $\mathfrak{s u}(2)$ factor, and $\left\{u_{4}, u_{5}, u_{6}\right\}$ span the other $\mathfrak{s u}(2)$ 
factor. In this basis, the map $\phi$ of equation (4.51) becomes

$$
\begin{aligned}
& \phi\left(e_{1} \wedge e_{2}\right)=\left(u_{1}-u_{4}\right) / 2 \\
& \phi\left(e_{1} \wedge e_{3}\right)=-\left(u_{2}+u_{5}\right) / 2 \\
& \phi\left(e_{1} \wedge e_{4}\right)=\left(u_{3}-u_{6}\right) / 2 \\
& \phi\left(e_{2} \wedge e_{3}\right)=\left(u_{3}+u_{6}\right) / 2 \\
& \phi\left(e_{2} \wedge e_{4}\right)=\left(u_{2}-u_{5}\right) / 2 \\
& \phi\left(e_{3} \wedge e_{4}\right)=\left(u_{1}+u_{4}\right) / 2
\end{aligned}
$$

Now let $u_{4}=u_{5}=0$ so that we are left with $\left\{u_{1}, u_{2}, u_{3}, u_{6}\right\}$ which forms a basis for $\mathfrak{s u}(2) \times \mathfrak{u}(1)$. Then a fundamental requirement for the LATKe commutator is violated:

$$
\left(\phi\left(v_{i} \wedge v_{j}\right)\right) \cdot v_{k} \neq-\left(\phi\left(v_{k} \wedge v_{j}\right)\right) \cdot v_{i} \quad \forall v_{i}, v_{j}, v_{k} \in V .
$$

For example,

$$
\phi\left(e_{1} \wedge e_{2}\right) \cdot e_{3}=\frac{u_{1}}{2} \cdot e_{3}=\frac{\left(e_{12}+e_{34}\right)}{2} \cdot e_{3}=\frac{e_{4}}{2}
$$

while

$$
\phi\left(e_{3} \wedge e_{2}\right) \cdot e_{1}=-\frac{\left(u_{3}+u_{6}\right)}{2} \cdot e_{3}=-e_{14} \cdot e_{1}=-e_{4},
$$

so $\phi\left(e_{1} \wedge e_{2}\right) \cdot e_{3} \neq-\phi\left(e_{3} \wedge e_{2}\right) \cdot e_{1}$. The same can be checked for other combinations of $e_{i}$.

So there is no longer a well-defined LATKe commutator and not even a sub-LATKe remains.

Another way of stating this result is that $\mathfrak{g}_{\mathfrak{L}}$ is unbreakable as long as $\mathfrak{L}$ is present; or, that $\mathfrak{L}$ protects $\mathfrak{g}_{\mathfrak{L}}$ from being broken. This unbreakability of $\mathfrak{g}_{\mathfrak{L}}$ may remind one of some global symmetries which may not be broken under certain conditions [26]. One may be tempted to interpret the LATKe to be a manifestation of these conditions. 


\section{Vacuum Selection Mechanism}

There was great excitement in the physics community in the 1980's when it was discovered, through a study of anomaly cancellation, that string theory came along with gauge groups - either $E_{8} \times E_{8}$ or $S O(32)$; this discovery allowed for the hope that string theory might have some applications to phenomenology, which is governed by gauge theories [27, 28].

In the decades that followed, a great number of attempts at engineering a Calabi-Yau or $G_{2}$ space were carried out with the purpose of obtaining a theory in four dimensions that is as close as possible to the standard model. As it happened, orbifolds were employed in Calabi-Yau compactifications of heterotic string theory for this purpose, since they induced gauge symmetry breaking by Wilson lines [29, 30, 31], making the gauge group closer to the standard model group. They also reduced the number of fermion generations that arise from the compactification, bringing that number closer to the phenomenological value of three.

Since then, it has been realized [32] that there is a staggering number of Calabi-Yau or $G_{2}$ spaces, making up what is called today the "string landscape". Therefore, the idea of a "vacuum selection mechanism," which is some principle that would single out one vacuum or at least narrow down the choices considerably, has been sought after.

The uniqueness of the LATKe is a vacuum selection mechanism. The selected compactification space is a Calabi-Yau or $G_{2}$ space with a $\mathbf{C}^{3} / \mathbf{Z}_{3}$ singularity, and the selected vacuum theory is a supersymmetric $\mathfrak{s u}(2) \times \mathfrak{s u}(2)$ gauge theory with matter in the $(2,2)$ representation.

While it has been accepted that no vacuum selection mechanisms have as yet been proposed [32], in retrospect we claim that before the present work, there did exist a vacuum selection mechanism: anomaly cancellation. It selected a string theory with gauge group either $E_{8} \times E_{8}$ or $S O(32)$.

While neither the uniqueness of the LATKe nor anomaly cancellation actually selects the standard model itself, our unique, simple LATKe Yang-Mills is tantalizingly close to the standard model. 
Acknowledgements I am deeply indebted to I. M. Singer for inspiration and advice during the various stages of this work and for reading and commenting on an earlier draft. I am deeply indebted to F. Wilczek for discussions, inspiration, nurturing, and mentoring. I wish to thank R. Jackiw, P. Deligne, V. Kac, M. Goresky, D. Kazhdan, R. MacPherson, D. Freedman, J. Harris, M. Artin, P. Kronheimer, X. de la Ossa, J. de Jong, R. L. Jaffe, B. Zwiebach, S. Steadman, E. Farhi, W. Taylor, and C. Vafa for helpful discussions. I am very grateful to Y.P. Lee for helpful discussions and for reading and commenting on a draft. Last, but not least, I am grateful to A. Wiles for hospitality and encouragement. This work was supported in part by funds provided by the U.S. Department of Energy (D.O.E.) under cooperative research agreement DE-FC02-94ER40818.

\section{A Blow-up of $\mathrm{C}^{n} / \mathbf{Z}_{n}$}

Let $\mathbf{Z}_{n}$ be the multiplicative group generated by $\epsilon_{n} I_{n}$, where $\epsilon_{n}=e^{2 \pi i / n}$ and $I_{n}$ is the $n \times n$ identity matrix. Let $\epsilon_{n} \in \mathbf{Z}_{n}$ act on $\left(z_{1}, \ldots, z_{n}\right) \in \mathbf{C}^{n}$ by

$$
\left(z_{1}, \ldots, z_{n}\right) \rightarrow\left(\epsilon_{n} z_{1}, \ldots, \epsilon_{n} z_{n}\right)
$$

Denote the equivalence classes in the quotient $X_{n}=\mathbf{C}^{n} / \mathbf{Z}_{n}$ by $\left[z_{1}, \ldots, z_{n}\right]$.

The origin of $\mathbf{C}^{n}$ is fixed under this action. The resolution of the singularity at the origin is given as follows.

Let $Y_{n}=\left(\mathbf{C}^{n+1}-\{0\}\right) / \mathbf{C}^{*}$, where $\lambda \in \mathbf{C}^{*}$ acts via

$$
\left(w_{1}, \ldots, w_{n}, w_{n+1}\right) \rightarrow\left(\lambda w_{1}, \ldots, \lambda w_{n}, \lambda^{-n} w_{n+1}\right)
$$

Denote equivalence classes in $Y_{n}$ by $\left[w_{1}, \ldots, w_{n}, w_{n+1}\right]$. Then $\pi: Y_{n} \rightarrow X_{n}$ is given by

$$
\begin{aligned}
\pi\left(\left[w_{1}, \ldots, w_{n}, 1\right]\right) & =\left[w_{1}, \ldots, w_{n}\right] \\
\pi\left(\left[w_{1}, \ldots, w_{n}, 0\right)\right. & =[0, \ldots, 0]
\end{aligned}
$$


Equation (A.3) is one-to-one: the equivalence class $\left[w_{1}, \ldots, w_{n}, 1\right] \in Y_{n}$ is determined by setting $w_{n+1}=1=\lambda^{-n} w_{n+1}$ so now the $\lambda$ appearing in equation (A.2) is any $n^{\text {th }}$ root of unity, leading to the same quotient action as the one defining $X_{n}$ in equation (A.1).

Equation (A.4) provides us with the exceptional divisor: $\pi^{-1}([0, \ldots, 0])$ is the set $\left[w_{1}, \ldots, w_{n}, 0\right]$, which is just $\mathbf{P}^{n-1}$ given the action in equation (A.2).

\section{B The LAnKe $\mathfrak{L}_{n}$}

Let $V_{n+1}$ be the standard $(n+1)$-dimensional vector representation of $\mathfrak{s o}(n+1)$, and let $e_{i j}$ and $e_{i}$ be defined as in equations (4.26) and (4.27). Generalizing equation (4.51), we define a map $\phi_{n}: \Lambda^{n-1} V_{n+1} \rightarrow \mathfrak{s o}(n+1)$ as follows:

$$
\phi_{n}\left(e_{1} \wedge \cdots \wedge \hat{e}_{i} \wedge \cdots \wedge \hat{e}_{j} \wedge \cdots \wedge e_{n+1}\right)=(-1)^{i+j+1} e_{i j},
$$

where a hat over $e_{i}$ means that it is omitted. This map yields a commutator of the $n$-th kind:

$$
\begin{aligned}
{\left[e_{1} \wedge \cdots \wedge \hat{e}_{i} \wedge \cdots \wedge e_{n}\right] } & =\phi_{n}\left(e_{1} \wedge \cdots \wedge \hat{e}_{i} \wedge \cdots \wedge e_{n-1}\right) \cdot e_{n} \\
& =(-1)^{i+n+1} e_{i n} \cdot e_{n}=(-1)^{i+n+1} e_{i}, i<n(\mathrm{~B},) \\
{\left[e_{1} \wedge \cdots \wedge e_{n-1}\right] } & =\phi_{n}\left(e_{1} \wedge \cdots \wedge e_{n-2}\right) \cdot e_{n-1} \\
& =(-1)^{(n-1)+n+1} e_{(n-1) n} \cdot e_{n}=-e_{n}
\end{aligned}
$$

It satisfies the requirements for a Lie algebra of the $n$-th kind (Definition 5.2).

After a change of variables, one can show that for $\mathfrak{L}_{n}$, there is a Cartan subalgebra $\mathfrak{h}_{\mathfrak{L}_{n}}$ of dimension $n-1$ so $\Lambda^{n-1} \mathfrak{h}_{\mathfrak{L}_{n}}$ is one dimensional and there is a one-dimensional root space, where a root is in the dual space of $\Lambda^{n-1} \mathfrak{h}_{\mathfrak{L}_{n}}$ :

$$
\alpha: \Lambda^{n-1} \mathfrak{h}_{\mathfrak{L}_{n}} \longrightarrow \mathbf{C}
$$

The Dynkin diagram of $\mathfrak{L}_{n}$ has one node corresponding to the single cycle $\mathbf{P}^{n-1}$ in the exceptional divisor of the singularity $\mathbf{C}^{n} / \mathbf{Z}_{n}$. 
All the mathematical definitions related to LATKes generalize quite naturally to LAnKes. In addition, LATKe Yang-Mills easily generalizes to LAnKe Yang-Mills, and for $\mathfrak{L}_{n}$, LAnKe Yang-Mills theory is an $\mathfrak{s o}(n+1)$ gauge theory with matter in the $(n+1)$-dimensional vector representation.

\section{The map $\omega$ for $B_{7}$}

Let $\left\{E_{m n}\right\}_{k l}=\delta_{m k} \delta_{n l}$ be $7 \times 7$ matrices. Then the following denotes a basis for $\mathfrak{s o}_{7}[20,33]$ :

$$
\begin{array}{ll}
\epsilon_{0 i}=E_{0 i}-E_{i+3,0} & (i=1,2,3) \\
\epsilon_{0, i+3}=E_{0, i+3}-E_{i, 0} & (i=1,2,3) \\
\mu_{i j}=E_{i j}-E_{j+3, i+3} & (i, j=1,2,3) \\
\nu_{i j}=E_{i, j+3}-E_{j, i+3} & (1 \leq i<j \leq 3) \\
\rho_{i j}=E_{i+3, j}-E_{j+3, i} & (1 \leq i<j \leq 3) .
\end{array}
$$

Then $H_{1}=\mu_{11}, H_{2}=\mu_{22}, H_{3}=\mu_{33}$ form the Cartan subalgebra. Recall the basis $\left\{e_{i}, e_{j+3}, e_{0}\right\}, i, j=1,2,3$ for $V_{7}$. The embedding $\psi \cdot \phi^{-1}$ of $\mathfrak{s o}_{7}$ in $C\left(V_{7}, Q\right)$ (Equation (4.38)) is given by

$$
\begin{aligned}
\epsilon_{0 i} & \mapsto \frac{1}{2} e_{0} e_{i+3} \\
\epsilon_{0, i+3} & \mapsto \frac{1}{2} e_{0} e_{i} \\
\mu_{i j} & \mapsto \frac{1}{2} e_{i} e_{j+3}-\frac{1}{2} \delta_{i j} \\
\nu_{i j} & \mapsto \frac{1}{2} e_{i} e_{j} \\
\rho_{i j} & \mapsto \frac{1}{2} e_{i+3} e_{j+3} .
\end{aligned}
$$

Let $f_{1}, \ldots, f_{8}$ be the basis for $\Lambda^{\bullet} W$ with $f_{1}=1, f_{2}=e_{1} \wedge e_{2}, f_{3}=e_{1} \wedge e_{3}$, $f_{4}=e_{2} \wedge e_{3}, f_{k}=e_{k-4}$ for $k=5,6,7$, and $f_{8}=e_{1} \wedge e_{2} \wedge e_{3}$. Then equation (4.47) becomes

$$
\omega\left(f_{1} \wedge f_{5}\right)=\rho_{23}
$$


Using the action of $\mathfrak{s o}_{7}$ on $\Lambda^{2}\left(\Lambda^{\bullet} W\right)$ together with the intertwining condition on $\omega$ then yields

$$
\begin{aligned}
& \omega\left(f_{1} \wedge f_{5}\right)=\rho_{23} ; \omega\left(f_{1} \wedge f_{6}\right)=-\rho_{13} ; \omega\left(f_{1} \wedge f_{7}\right)=\rho_{12} ; \\
& \omega\left(f_{2} \wedge f_{5}\right)=2 \mu_{13} ; \omega\left(f_{2} \wedge f_{6}\right)=2 \mu_{23} ; \omega\left(f_{2} \wedge f_{8}\right)=4 \nu_{12} ; \\
& \omega\left(f_{3} \wedge f_{5}\right)=-2 \mu_{12} ; \omega\left(f_{3} \wedge f_{7}\right)=-2 \mu_{32} ; \omega\left(f_{3} \wedge f_{8}\right)=4 \nu_{13} ; \\
& \omega\left(f_{4} \wedge f_{6}\right)=2 \mu_{21} ; \omega\left(f_{4} \wedge f_{7}\right)=2 \mu_{31} ; \omega\left(f_{4} \wedge f_{8}\right)=4 \nu_{23} ; \\
& \omega\left(f_{1} \wedge f_{2}\right)=-\epsilon_{03} ; \omega\left(f_{1} \wedge f_{3}\right)=\epsilon_{02} ; \omega\left(f_{1} \wedge f_{4}\right)=-\epsilon_{01} \\
& \omega\left(f_{2} \wedge f_{3}\right)=2 \epsilon_{04} ; \omega\left(f_{2} \wedge f_{4}\right)=2 \epsilon_{05} ; \omega\left(f_{3} \wedge f_{4}\right)=2 \epsilon_{06} ; \\
& \omega\left(f_{5} \wedge f_{6}\right)=\epsilon_{03} ; \omega\left(f_{5} \wedge f_{7}\right)=-\epsilon_{02} ; \omega\left(f_{5} \wedge f_{8}\right)=-2 \epsilon_{04} ; \\
& \omega\left(f_{6} \wedge f_{7}\right)=\epsilon_{01} ; \omega\left(f_{6} \wedge f_{8}\right)=-2 \epsilon_{05} ; \omega\left(f_{7} \wedge f_{8}\right)=-2 \epsilon_{06} ; \\
& \omega\left(f_{1} \wedge f_{8}\right)=-\mu_{11}-\mu_{22}-\mu_{33} ; \omega\left(f_{2} \wedge f_{7}\right)=-\mu_{11}-\mu_{22}+\mu_{33} \\
& \omega\left(f_{4} \wedge f_{5}\right)=\mu_{11}-\mu_{22}-\mu_{33} ; \omega\left(f_{3} \wedge f_{6}\right)=\mu_{11}-\mu_{22}+\mu_{33}
\end{aligned}
$$

Note added: Refs. [34]-[39] were brought to the authors attention after this work was completed and posted.

\section{References}

[1] C. M. Hull and P. K. Townsend, "Enhanced gauge symmetries in superstring theories," Nucl. Phys. B 451, 525 (1995) [arXiv:hep-th/9505073].

[2] E. Witten, "String theory dynamics in various dimensions," Nucl. Phys. B 443, 85 (1995) [arXiv:hep-th/9503124].

[3] B. S. Acharya, "M theory, Joyce orbifolds and super Yang-Mills," Adv. Theor. Math. Phys. 3, 227 (1999) [arXiv:hep-th/9812205].

[4] B. S. Acharya, "On realising $\mathrm{N}=1$ super Yang-Mills in M theory," arXiv:hep-th/0011089.

[5] M. Atiyah and E. Witten, "M-theory dynamics on a manifold of $G_{2}$ holonomy," Adv. Theor. Math. Phys. 6, 1 (2003) [arXiv:hep-th/0107177]. 
[6] H. Georgi and S. L. Glashow, "Unity of all elementary particle forces," Phys. Rev. Lett. 32, 438 (1974).

[7] T. Friedmann, "On the quantum moduli space of M theory compactifications," Nucl. Phys. B 635, 384 (2002) [arXiv:hep-th/0203256].

[8] T. Friedmann, "Physics through extra dimensions: on dualities, unification, and pair production," Princeton University, PhD thesis (2003), UMI-31-03026; http://www.princeton.edu/physics/academics/graduateprogram/theses/theses-from-2003/T.Friedmannthesis.pdf

[9] T. Friedmann and E. Witten, "Unification scale, proton decay, and manifolds of $G_{2}$ holonomy," Adv. Theor. Math. Phys. 7, 577 (2003) [arXiv:hepth/0211269].

[10] P. Du Val, "On isolated singularities of surfaces which do not affect the conditions of adjunctions," Proc. Cambridge Philos. Soc. 30, 453-465; 483-491 (1933/34).

[11] M. Artin, "On isolated rational singularities of surfaces," Amer. J. Math. 88 129-136 (1966).

[12] R. Steinberg, "Kleinian singularities and unipotent elements," Proc. Sympos. Pure Math., 37 (1980) 265.

[13] F. Klein, "Lectures on the ikosahedron and the solution of equations of the fifth degree," translated by G.G. Morrice. London: Trubner \& Co., 1888 .

[14] P. Mayr, "Geometric construction of $\mathrm{N}=2$ gauge theories," Fortsch. Phys. 47, 39 (1999) [arXiv:hep-th/9807096].

[15] S. H. Katz, A. Klemm and C. Vafa, "Geometric engineering of quantum field theories," Nucl. Phys. B 497, 173 (1997) [arXiv:hep-th/9609239].

[16] C. Vafa, private communication.

[17] S. S. Y. Yau and Y. Yu, "Gorenstein quotient singularities in dimension three," Mem. Amer. Math. Soc. 105, 505 (1993).

[18] V. G. Kac, "Lie superalgebras," Adv. in Math. 26, 8 (1977). 
[19] J. C. Baez, "The Octonions," Bull. Am. Math. Soc. 39, 145 (2001). arXiv:math/0105155.

[20] W. Fulton and J. Harris, "Representation Theory: A First Course," Springer, New York, 1991.

[21] R. D. Schafer, "Introduction to Non-Associative Algebras," Academic Press, New York, 1966.

[22] H. Georgi, "Lie algebras in particle physics. From isospin to unified theories," Front. Phys. 54, 1 (1982).

[23] C. N. Yang and R. L. Mills, "Conservation of isotopic spin and isotopic gauge invariance," Phys. Rev. 96, 191 (1954).

[24] R. Bryant and S. Salamon, "On the construction of some complete metrics with exceptional holonomy," Duke Math. J. 58 (1989) 829.

[25] G. W. Gibbons, D. N. Page and C. N. Pope, "Einstein metrics on $S^{3}, R^{3}$ and $R^{4}$ bundles," Commun. Math. Phys. 127, 529 (1990).

[26] C. Vafa and E. Witten, "Restrictions on symmetry breaking in vector-like gauge theories," Nucl. Phys. B 234, 173 (1984).

[27] M. B. Green and J. H. Schwarz, "Anomaly cancellation in supersymmetric $\mathrm{D}=10$ gauge theory and superstring theory," Phys. Lett. B 149, 117 (1984).

[28] D. J. Gross, J. A. Harvey, E. J. Martinec and R. Rohm, "The heterotic string," Phys. Rev. Lett. 54, 502 (1985).

[29] Y. Hosotani, "Dynamical mass generation by compact extra dimensions," Phys. Lett. B 126, 309 (1983).

[30] Y. Hosotani, "Dynamical gauge symmetry breaking as the Casimir effect," Phys. Lett. B 129, 193 (1983).

[31] P. Candelas, G. T. Horowitz, A. Strominger and E. Witten, "Vacuum configurations for superstrings," Nucl. Phys. B 258, 46 (1985).

[32] M. R. Douglas, "The statistics of string / M theory vacua," JHEP 0305, 046 (2003) [arXiv:hep-th/0303194]. 
[33] G. G. A. Bauerle and E. A. de Kerf, "Lie Algebras. Finite and Infinite Dimensional Lie Algebras and Applications in Physics," North-Holland, Amsterdam, 1990.

[34] V. Filipov, "n-Lie algebras," Sibirsk. Math. Zh. 26, 126 (1985); S. M. Kasymov, "On a theory of n-Lie algebras," Algebra i Logika 26, 277 (1987).

[35] Y. Nambu, "Generalized Hamiltonian dynamics," Phys. Rev. D 7, 2405 (1973).

[36] J. Bagger and N. Lambert, "Three-Algebras and N=6 Chern-Simons Gauge Theories," Phys. Rev. D 79, 025002 (2009) [arXiv:0807.0163 [hep-th]]; A. Gustavsson, "Algebraic structures on parallel M2-branes," Nucl. Phys. B 811, 66 (2009) [arXiv:0709.1260 [hep-th]]; J. Bagger and N. Lambert, "Gauge Symmetry and Supersymmetry of Multiple M2-Branes," Phys. Rev. D 77, 065008 (2008) [arXiv:0711.0955 [hepth]]; P. De Medeiros, J. M. Figueroa-O'Farrill and E. Mendez-Escobar, "Lorentzian Lie 3-algebras and their Bagger-Lambert moduli space," JHEP 0807, 111 (2008) [arXiv:0805.4363 [hep-th]].

[37] W. X. Ling, "On the structure of n-Lie algebras," PhD Thesis, Siegen, 1993, unpublished.

[38] A. S. Dzhumadil'daev, "Representations of vector product n-Lie algebras," Comm. Algebra, 32, 3315 (2004); A. S. Dzhumadil'daev, "Identities and derivations for jacobian algebras," Contemp. Math J. 315, 245 (2002).

[39] L. A. Takhtajan, "On foundation of the generalized Nambu mechanics," Comm. Math. Phys. 160, 295 (1994); Yu. L. Daletskii and L. A. Takhtajan, "Leibniz and Lie algebra structures for Nambu algebra," Lett. Math. Phys. 39127141 (1997). 